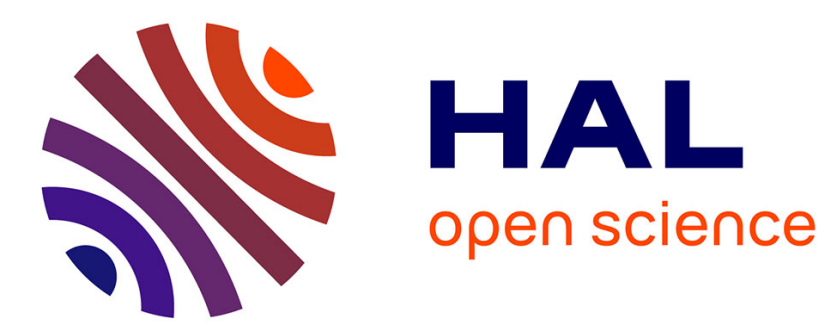

\title{
Construction and stepwise refinement of dependability models
}

\author{
Claudia Betous-Almeida, Karama Kanoun
}

\section{To cite this version:}

Claudia Betous-Almeida, Karama Kanoun. Construction and stepwise refinement of dependability models. Performance Evaluation, 2004, 56 (1-4), pp.277-306. hal-01980966

\section{HAL Id: hal-01980966 https://hal.science/hal-01980966}

Submitted on 5 Feb 2019

HAL is a multi-disciplinary open access archive for the deposit and dissemination of scientific research documents, whether they are published or not. The documents may come from teaching and research institutions in France or abroad, or from public or private research centers.
L'archive ouverte pluridisciplinaire HAL, est destinée au dépôt et à la diffusion de documents scientifiques de niveau recherche, publiés ou non, émanant des établissements d'enseignement et de recherche français ou étrangers, des laboratoires publics ou privés. 


\title{
Construction and Stepwise Refinement
}

\section{of Dependability Models}

\author{
Cláudia Betous-Almeida and Karama Kanoun \\ LAAS-CNRS \\ 7, Avenue du Colonel Roche \\ 31077 Toulouse Cedex 4 - France \\ \{almeida, kanoun\}elaas. fr
}

\begin{abstract}
This paper presents a stepwise approach for dependability modeling, based on Generalized Stochas tic Petri Nets (GSPNs). The first-step model called functional-level model, is built based on the system functional specifications and then completed by the structural model as soon as the system's architecture is known. It can then be refined according to three complementary aspects: Component decomposition, state and event finetuning and distribution adjustment to take into account increasing event rates. We define specific rules to make the successive transformations as easy and systematic as possible. This approach allows the various dependencies to be taken into account at the right level of abstraction: Functional dependency, structural dependency and those induced by non-exponential distributions. A part of the approach is applied to an instrumentation and control system $(I \& C)$ in power plants.
\end{abstract}

\section{Introduction}

Dependability evaluation plays an important role in critical systems' definition, design and development. Modeling can start as early as system functional specifications, from which a funtional-level model can be derived to help in analyzing dependencies between the various functions. This model can then be completed and refined by incorporating more information about the system's structure, including dependencies between system's components.

The starting point of our work was to help (based on dependability evaluation) a stakeholder of an I\&C system in selecting and refining systems proposed by various contractors in response to a Call for Tenders. To this end, we have defined a stepwise modeling approach that can be easily used to select an appropriate system and to model it thoroughly. This modeling approach is general and can be applied to any system, to model its dependability in a progressive way. Thus, it can be used by any system's developer. 
The process of defining and implementing an I\&C system can be viewed as a multi-phase process starting from the issue of a call for tenders by the stakeholder. The call for tenders gives the functional and non-functional (e.g., dependability) requirements of the system and asks candidate contractors to make offers for possible systems/architectures satisfying the specified requirements. A preliminary analysis of the numerous responses by the stakeholder, according to specific criteria, allows the pre-selection of two or three candidate systems. At this stage, the candidate systems are defined at a high level and the application software is not entirely written. The comparative analysis of the pre-selected candidate systems, in a second step, allows the selection of the most appropriate one. Finally, the retained system is refined and thoroughly analyzed to go through qualification. This process is illustrated in Fig. 1. Even though this process is specific to a given company, the various phases are similar to those of a large category of critical systems.

Dependability modeling and evaluation constitute an efficient support for the selection and refinement processes, thorough analysis and preparation for the system's qualification. Our modeling approach follows the same steps as the development process. It is performed in three steps as described in Fig. 1 :

Step 1. Construction of a functional-level model based on the system's specifications;

Step 2. Transformation of the functional-level model into a high-level dependability model, based on the knowledge of the system's structure. A model is generated for each pre-selected candidate system, the aim being the comparaison of the pre-selected systems;

Step 3. For the selected system, refinement of the high-level model into a detailed dependability model.

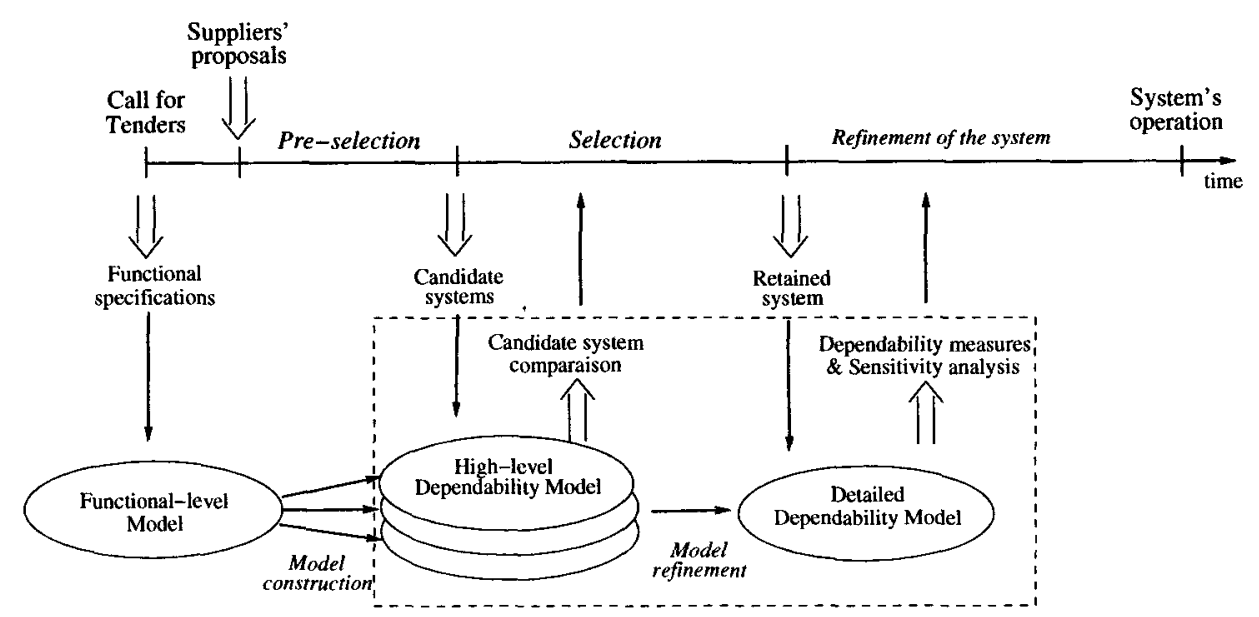

Fig. 1. Various steps of $I \& C$ definition and implementation, and modeling 
Modeling is based on Generalized Stochastic Petri Nets (GSPN) [2] due to their ability to cope with modularity and model refinement. The GSPN model is processed to obtain the associated dependability measures (i.e., availability, reliability, safety, ...) using an evaluation tool such as SURF-2 [7].

The relevance of our approach lies in supplying a set of coherent techniques, allowing to master step by step dependability model construction, based on GSPNs. It allows the progressive incorporation of the newly available information into the existing model, changing its initial organization according to a well identified set of rules. Model refinement can be achieved to take into account: Component decomposition, state/event fine-tuning and distribution adjustment. In particular, the same set of rules is used for generating the high-level model from the functional-level model and for component refinement. We have adapted the method of stages (used for simulating increasing failure rates) to take into account dependencies between interacting components without changing their initial models.

This modeling approach has been applied to three different I\&C systems, to help select the most appropriate one [6]. In this paper we illustrate our approach on a small part of one of them. This paper is an elaboration of our previous work [3,6], and an extension of [5], to which we have added the construction rules' formalization and detailed the application in Section 5. [3] was devoted only to the high-level dependability model's construction from the functional-level and did not address at all the structural model's refinement. [6] mainly refers to the comparison of the three different I\&C systems at a high-level.

The remainder of the paper is organized as follows. Section 2 describes the functional-level model. The high-level dependability model construction is presented in Section 3. Section 4 deals with the structural model's refinement and Section 5 presents an example of application of the proposed approach to an I\&C system. Finally, Section 6 concludes the paper.

\section{Functional-level Model}

The derivation of the system's functional-level model is the first step of our method. This model is independent of the underlying system's structure. Hence, it can be built even before the call for tenders, by the stakeholder. It is formed by places representing possible states of functions. For each function, the minimal number of places is two (Fig. 2): One represents the function's nominal state (F) and the other its failure state $(\overline{\mathrm{F}})$.

In the following, we assume only one failure mode, but it is applicable in the same manner when there are several failure modes per function. Between states $F$ and $\overline{\mathrm{F}}$, there are events that manage changes from 


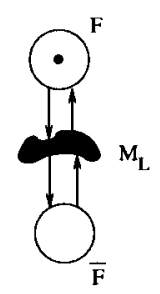

Fig. 2. Functional-level model related to a single function

F to $\bar{F}$ and vice-versa. These events are inherent to the system's structure that is not specified at this step, as it is not known yet. The model containing these events and the corresponding places, is called the link model $\left(\mathrm{M}_{\mathrm{L}}\right)$. Note that the set $\left\{\mathrm{F}, \mathrm{M}_{\mathrm{L}}, \overline{\mathrm{F}}\right\}$, that constitutes the system's GSPN model, will be completed once the system's structure is known.

However, systems generally perform more than one function. In this case we have to look for dependencies between these functions due to the communication between them. We distinguish two degrees of dependency: Total dependency and partial dependency.

Case (a) Total dependency $-F_{2}$ depends totally on $F_{1}$ (noted $F_{2} \hookleftarrow F_{1}$ ): If $F_{1}$ fails, $F_{2}$ also fails. This means that the probability that $F_{2}$ fails equals the probability that $F_{1}$ fails, times the probability that $F_{2}$ fails due to the failure of its components;

Case (b) Partial dependency $-F_{2}$ depends partially on $F_{1}$ (noted $F_{2} \hookleftarrow F_{1}$ ): $F_{1}$ 's failure does not induce $F_{2}$ 's failure, but it puts $F_{2}$ in a degraded state. In Fig. 3, the degraded state is represented by place $F_{2 d}$ that is marked whenever $F_{1}$ is in its failure state and $F_{2}$ in its nominal one. The token is removed from $F_{2 d}$ as soon as $F_{1}$ returns to its nominal state, however other scenarios might be considered.

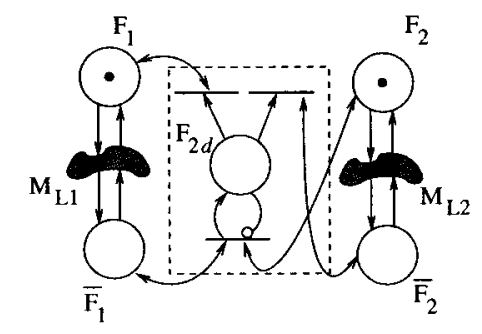

Fig. 3. Partial functional dependency $\left(\mathrm{F}_{2} \hookleftarrow \mathrm{F}_{1}\right)$ 


\section{High-level Dependability Model}

The high-level dependability model is formed by the function's states and the link model that gathers the set of states and events related to the system's structural behavior. This behavior is modeled by the so-called structural model $\left(\mathrm{M}_{\mathrm{S}}\right)$ and then it is connected to $\mathrm{F}$ and $\overrightarrow{\mathrm{F}}$ places through an interface model $\left(\mathrm{M}_{\mathrm{I}}\right)$. The link model is thus made up of the structural model and of the interface model.

The structural model represents the behavior of the hardware and software components taking into account fault-tolerance mechanisms, maintenance policies as well as dependencies due to the interactions between components.

The interface model connects the structural model with its functional state places by a set of immediate transitions.

In this section, we mainly concentrate on the interface model. In particular, we assume that the structural model can be built by applying one of the many existing modular modeling approaches (see e.g., $[8,12-14])$, and we focus on its refinement in Section 4 . Note that the structural models presented in this section are not complete, some examples of complete structural models are given in Section 4 . We present simple examples to help understand the notion of interface model before presenting the general interfacing rules.

\subsection{Examples of Interface Models}

For sake of simplicity, we first consider the case of a single function then the case of multiple functions.

Single Function Several situations may be taken into account. Since the two most important cases are the series and the combination series-parallel components, we limit the illustrations to these two basic cases which allow modeling of any system. More details are given in $[3,4]$.

- Series case: Suppose function F carried out by a software component $\mathrm{S}$ and a hardware component $\mathrm{H}$. Then, $\mathrm{F}$ and $\overline{\mathrm{F}}$ places' markings depend upon the markings of the hardware and software components models (Fig. 4).

The behavior of $\mathrm{H}$ and $\mathrm{S}$ is modeled by the structural model and then it is connected to places $\mathrm{F}$ and $\bar{F}$ through an interface model. Note that there is only one interface model. We split it into two parts, an upstream part and a downstream part, so that it is constructed in a systematic way. This allows our approach to be re-usable, facilitating the construction of several models related to various architectures. Also, the case of simultaneous failures is not treated at this level. 


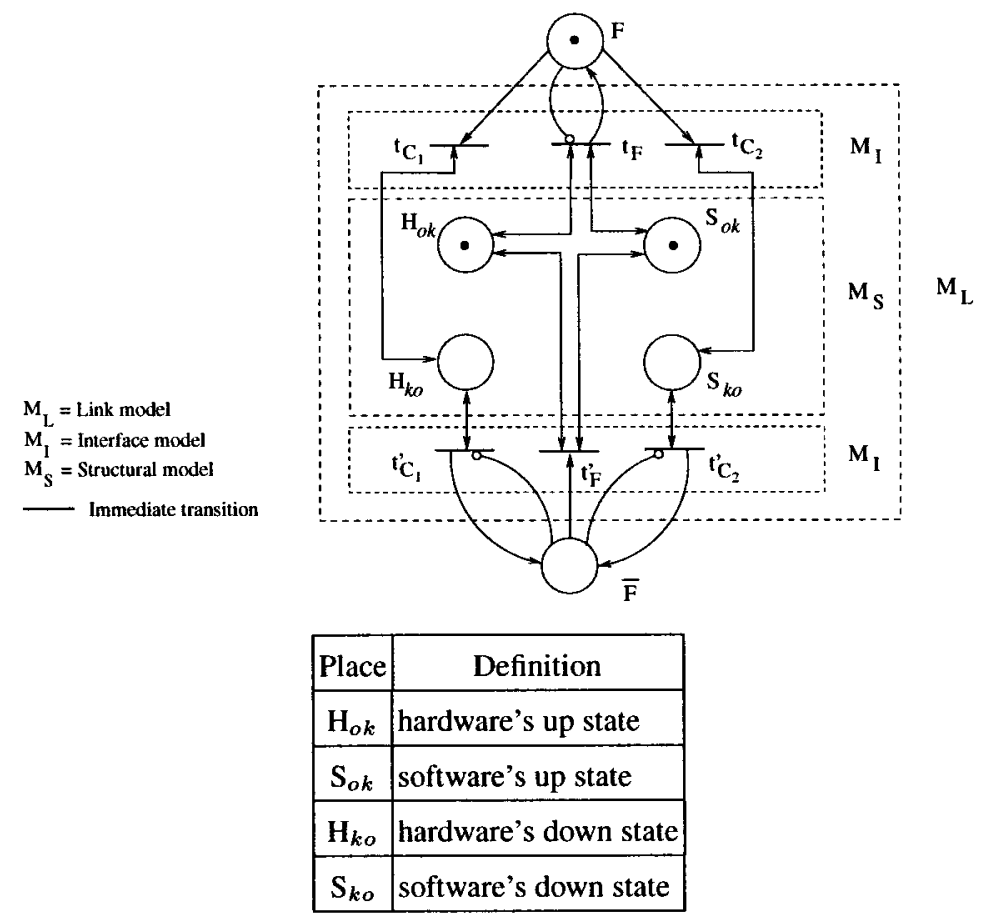

Fig. 4. Two series components

- Series-parallel case: Consider function F implemented by two redundant software components $\mathrm{S}_{1}$ and $S_{2}$, running on the same hardware component $\mathrm{H}$. F's up state is the combined result of H's up state and $S_{1}$ or $S_{2}$ 's up states, and F's failure state is the result of $H$ 's failure or $S_{1}$ and $S_{2}$ 's failure, as indicated in Fig. 5.

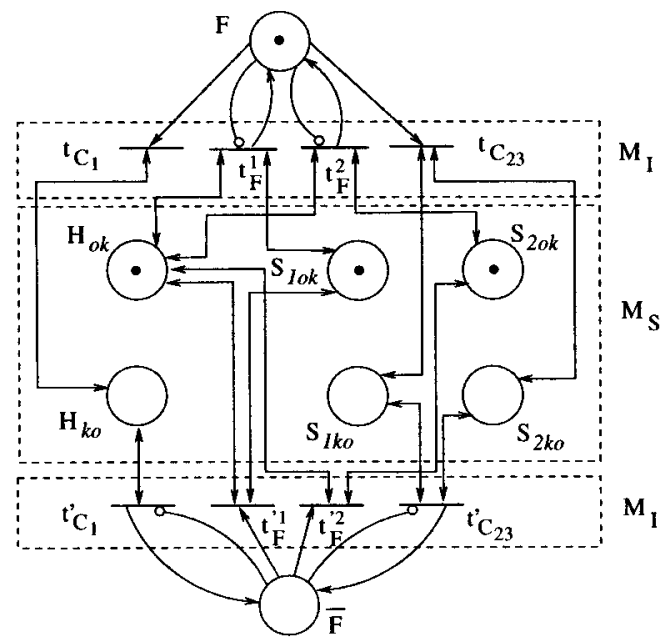

Fig. 5. Two redundant software components on a hardware component 
Multiple Functions Consider two functions (the generalization is straightforward) and let $\left\{\mathrm{C}_{1 \mathrm{i}}\right\}$ (resp. $\left\{C_{2 j}\right\}$ ) be the set of components associated to $F_{1}\left(\right.$ resp. $F_{2}$ ). We distinguish the case where functions do not share resources (such as components or repairmen), from the case where they share some. Examples of these two cases are presented hereafter.

- $F_{1}$ and $F_{2}$ have no common components: $\left\{\mathrm{C}_{1 \mathrm{i}}\right\} \cap\left\{\mathrm{C}_{2 \mathrm{j}}\right\}=\emptyset$. The interface models related to $\mathrm{F}_{1}$ and $F_{2}$ are built separately in the same way as explained for a single function. There are no structural dependencies, only functional ones.

- $F_{1}$ and $F_{2}$ have some common components: $\left\{\mathrm{C}_{1 \mathrm{i}}\right\} \cap\left\{\mathrm{C}_{2 \mathrm{j}}\right\} \neq \emptyset$. This corresponds to the existence of structural dependencies, in addition to functional dependencies. This case is illustrated on a simple example:

- $\mathrm{F}_{1}$ performed by three components: A hardware component $\mathrm{H}$ and two redundant software components $S_{11}$ and $S_{12}$. $F_{1}$ 's model corresponds to Fig. 5.

- $F_{2}$ performed by two components: The same hardware component $H$ as for $F_{1}$ and a software component $S_{21} . F_{2}$ 's model corresponds to Fig. 4 .

The global model of $F_{1}$ and $F_{2}$ is given in Fig. 6 . It can be seen that $\left.i\right)$ both interface models $\left(M_{I 1}\right.$ and $\mathrm{M}_{\mathrm{I} 2}$ ) are built separately, and ii) in the global model, the common hardware component $\mathrm{H}$ is represented only once by a common component model. Sharing of $\mathrm{H}$ thus creates a structural dependency. The functional dependencies are not represented in this figure.

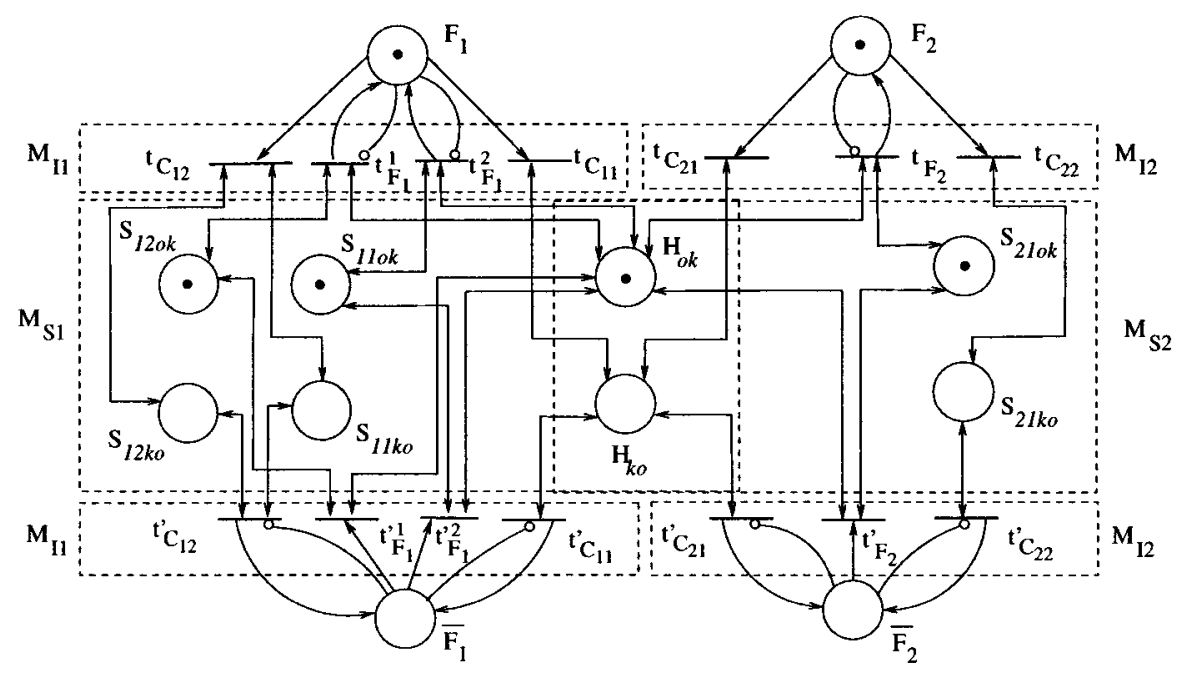

Fig. 6. Two functions with a structural dependency 


\subsection{Interfacing Rules}

The interface model $\mathrm{M}_{\mathrm{I}}$ connects the system's components with their functions by a set of transitions. This model is a key element in our approach. Particular examples of interface models have been given in Fig. 4 to 6 . In this section, the general organization of the interface model is presented. Interfacing rules are defined in formal terms after presenting them in an informal manner.

Overview of Interfacing Rules Upstream and downstream $M_{I}$ have the same number of immediate transitions and the arcs that are connected to these transitions are built in a systematic way:

- Upstream $M_{I}$ : It contains one function transition $\mathrm{t}_{\mathrm{F}}^{\mathrm{i}}$ for each series (set of) component(s), to mark the function's up state place, and one component transition $t_{C x}$ for each series, distinct component that has a direct impact on the functional model, to unmark the function's up state place.

- Each $\mathrm{t}_{\mathrm{F}}^{\mathrm{i}}$ is linked by an inhibitor arc to the function's up state place, by an arc to the function's up state place and by one bidirectional arc to each initial (ok) component's place;

- Each $t_{C x}$ is linked by an arc to the function's up state place and by one bidirectional arc to each failure component's place.

- Downstream $M_{I}$ : It contains one function transition $\mathrm{t}_{\mathrm{F}}^{\mathrm{i}}$ for each series (set of) component(s), to unmark the function's failure state place, and one component transition t' $\mathrm{Cx}$ for each series, distinct component that has a direct impact on the functional model, to mark the function's failure state place.

- Each $\mathrm{t}_{\mathrm{F}}^{\mathrm{i}}$ is linked by an arc to the function's failure state place and by one bidirectional arc to each initial (ok) component's place;

- Each $\mathbf{t}_{\mathrm{Cx}}$ is linked by an inhibitor arc to the function's failure state place, by an arc from the function's failure state place and by one bidirectional arc to each component's failure place.

Formal Definition of Interfacing Rules The interface model $\mathrm{M}_{\mathrm{I}}$ has been defined in formal terms. Its formal definition is presented hereafter:

- Transitions of $\mathbf{M}_{\mathbf{I}}$

Consider $T_{u p}$ (respectively $T_{\text {down }}$ ) the set of immediate transitions $t$ (respectively $t^{\prime}$ ) of the upstream (respectively downstream) part of the interface model, that is,

$$
T_{u p}=\left\{t: t \in \mathrm{M}_{\mathrm{I}} \mathrm{up}\right\} \text { and } T_{\text {down }}=\left\{t^{\prime}: t^{\prime} \in \mathrm{M}_{\mathrm{I}} \text { down }\right\}
$$


and $P$ (respectively $P^{\prime}$ ) the set of ok places (resp. ko places) of the structural model, that is,

$$
P=\left\{p_{o k}: p_{o k} \in \mathrm{M}_{\mathrm{S}}\right\} \text { and } P^{\prime}=\left\{p_{k o}: p_{k o} \in \mathrm{M}_{\mathrm{S}}\right\}
$$

Also,

- if $\mathrm{N}$ is the total number of components (corresponding equally to the number of tokens of $\mathrm{M}_{\mathrm{S}}$ 's initial marking), and

- if $\mathrm{Q}$ is the number of places having an initial marking not nil $(\mathrm{Q} \leq \mathrm{N}$ and $\mathrm{Q}=\operatorname{Card}(P))$ then,

$$
\operatorname{Card}\left(T_{u p}\right)=\operatorname{Card}\left(P^{\prime}\right)+1 \wedge \operatorname{Card}\left(T_{\text {down }}\right)=\operatorname{Card}(P)+1
$$

Plus, since by principle, $\operatorname{Card}(\mathrm{P})=\operatorname{Card}\left(\mathrm{P}^{\prime}\right)$

$$
\operatorname{Card}\left(T_{u p}\right)=\operatorname{Card}\left(T_{\text {down }}\right)
$$

- Arcs of $\mathrm{M}_{\mathrm{I}}$

Consider,

$-{ }^{\bullet} t$ corresponding to the set of input arcs of $t$ : Represented by the set of input places of $t$;

$-t^{\bullet}$ corresponding to the set of output arcs of $\mathrm{t}$ : Represented by the set of output places of $t$;

$-{ }^{\circ} t$ corresponding to the set of inhibitor arcs of $\mathrm{t}$ : Represented by the set of input places of $t$.

i. $\operatorname{Arcs}$ of $\mathrm{M}_{\mathrm{I}} u p$ :

$$
\begin{aligned}
& { }^{\bullet} t_{\mathrm{F}}=\left\{p_{x \mathrm{ok}}, p_{y \mathrm{ok}}: x \text { and } y \text { are in series }\right\} \\
& t_{\mathrm{F}}^{\bullet}=\{F\} \cup\left\{p_{x \mathrm{ok}}, p_{y \mathrm{ok}}: x \text { and } y \text { are in series }\right\} \\
& { }^{\circ} t_{\mathrm{F}}=\{F\} \\
& { }^{\bullet} t_{\mathrm{Cx}}=\{F\} \cup\left\{p_{x \mathrm{ko}}, p_{y \mathrm{ko}}: x \text { and } y \text { are in parallel }\right\} \\
& t^{\cdot} \dot{\mathrm{Cx}}_{\mathrm{x}}=\left\{p_{x \mathrm{ko}}, p_{y \mathrm{ko}}: x \text { and } y \text { are in parallel }\right\} \\
& { }^{\circ} t_{\mathrm{Cx}}=\emptyset
\end{aligned}
$$


ii. Arcs of $\mathrm{M}_{\mathrm{I}}$ down :

$$
\begin{aligned}
& \bullet t_{\mathrm{F}}^{\prime}=\{\overline{\mathrm{F}}\} \cup\left\{p_{x \mathrm{ok}}, p_{y \mathrm{ok}}: x \text { and } y \text { are in series }\right\} \\
& t^{\prime} \cdot p_{\mathrm{F}}=\left\{p_{x \mathrm{k}}, p_{y \mathrm{ok}}: x \text { and } y \text { are in series }\right\} \\
& { }^{\circ} t_{\mathrm{F}}^{\prime}=\emptyset \\
& \cdot t_{\mathrm{Cx}}^{\prime}=\left\{p_{x \mathrm{ko}}, p_{y \mathrm{ko}}: x \text { and } y \text { are in parallel }\right\} \\
& t_{\mathrm{C}}^{\prime}=\{\overline{\mathrm{F}}\} \cup\left\{p_{x \mathrm{ko}}, p_{y \mathrm{ko}}: x \text { and } y \text { are in parallel }\right\} \\
& { }^{\circ} t^{\prime}{ }_{\mathrm{Cx}}=\{\overline{\mathrm{F}}\}
\end{aligned}
$$

Rules have been presented for two components, nevertheless they can easily be generalised for several components.

- Weight of the arcs

It is given for the minimum marking of each place needed for the activation of the respective transition:

i. If components are all different:

a. They are all in series:

$$
\begin{aligned}
t_{\mathrm{F}}: \mathcal{M}(\mathrm{F})=0 & \wedge \forall_{x \leq N} \mathcal{M}\left(p_{x \mathrm{ok}}\right)=1 \\
t_{\mathrm{Cx}}: \mathcal{M}(\mathrm{F})=1 & \wedge \mathcal{M}\left(p_{x \mathrm{ko}}\right)=1 \\
t_{\mathrm{F}}^{\prime}: \mathcal{M}(\overline{\mathrm{F}})=1 & \wedge \forall_{x \leq N} \mathcal{M}\left(p_{x \mathrm{ok}}\right)=1 \\
t_{\mathrm{Cx}}^{\prime}: \mathcal{M}(\overline{\mathrm{F}})=0 & \wedge \mathcal{M}\left(p_{x \mathrm{ko}}\right)=1
\end{aligned}
$$

where $\mathrm{N}$ is the total number of components;

b. They are all in parallel:

$$
\begin{aligned}
& t_{\mathrm{F}}^{\mathrm{i}}: \mathcal{M}(\mathrm{F})=0 \wedge \mathcal{M}\left(p_{\text {iok }}\right)=1 \\
& t_{\mathrm{Cx}}: \mathcal{M}(\mathrm{F})=1 \wedge \forall_{x, y \leq N} \mathcal{M}\left(p_{x \mathrm{ko}}\right)=1 \wedge \mathcal{M}\left(p_{y \mathrm{ko}}\right)=1 \\
& t^{\prime \mathrm{i}} \mathrm{F}: \mathcal{M}(\overline{\mathrm{F}})=1 \wedge \mathcal{M}\left(p_{\text {iok }}\right)=1 \\
& t_{\mathrm{Cx}}^{\prime}: \mathcal{M}(\overline{\mathrm{F}})=0 \wedge\left(\forall_{x, y \leq N} \mathcal{M}\left(p_{\text {xko }}\right)=1 \vee \mathcal{M}\left(p_{y \mathrm{ko}}\right)=1\right)
\end{aligned}
$$


c. There are $Q$ in parallel and $R$ in series $(R+Q=N)$ :

$$
\begin{array}{r}
t_{\mathrm{F}}^{\mathrm{i}}: \mathcal{M}(\mathrm{F})=0 \wedge \forall_{x, y} \mathcal{M}\left(p_{x \mathrm{ok}}\right)=1 \wedge \mathcal{M}\left(p_{y \mathrm{ok}}\right)=1, \\
\mathrm{x} \text { and y are in series and } \mathrm{i}=1, \ldots, Q \\
t_{\mathrm{Cx}}: \mathcal{M}(\mathrm{F})=1 \wedge \forall_{x, y} \mathcal{M}\left(p_{x \mathrm{ko}}\right)=1 \wedge \mathcal{M}\left(p_{y \mathrm{ko}}\right)=1, \\
\mathrm{x} \text { and y are in parallel and } x=1, \ldots, R \\
t^{\prime \mathrm{i}}: \mathcal{M}(\overline{\mathrm{F}})=1 \wedge \forall_{x, y} \mathcal{M}\left(p_{x \mathrm{ok}}\right)=1 \wedge \mathcal{M}\left(p_{y \mathrm{ok}}\right)=1, \\
\mathrm{x} \text { and y are in parallel and } \mathrm{i}=1, \ldots, R \\
t_{\mathrm{Cx}}^{\prime}: \mathcal{M}(\overline{\mathrm{F}})=0 \wedge \forall_{x, y} \mathcal{M}\left(p_{x \mathrm{ko}}\right)=1 \wedge \mathcal{M}\left(p_{y \mathrm{ko}}\right)=1, \\
\mathrm{x} \text { and y are in series and } x=1, \ldots, Q
\end{array}
$$

ii. If there are some identical components and considering $\mathrm{r}$ the maximum number of tokens in place $p_{x}$ and $Q$ the number of ok places of $M_{S}(Q<N)$ :

a. They are all in series:

$$
\begin{aligned}
t_{\mathrm{F}}: \mathcal{M}(\mathrm{F})=0 & \wedge \forall_{x \leq Q} \mathcal{M}\left(p_{x \mathrm{ok}}\right)=r \\
t_{\mathrm{Cx}}: \mathcal{M}(\mathrm{F})=1 & \wedge \forall_{x \leq Q} \mathcal{M}\left(p_{x \mathrm{ko}}\right)=1 \\
t_{\mathrm{F}}^{\prime}: \mathcal{M}(\overline{\mathrm{F}})=1 & \wedge \forall_{x \leq Q} \mathcal{M}\left(p_{x \mathrm{ok}}\right)=r \\
t_{\mathrm{Cx}}^{\prime}: \mathcal{M}(\overline{\mathrm{F}})=0 & \wedge \forall_{x \leq Q} \mathcal{M}\left(p_{x \mathrm{ko}}\right)=1
\end{aligned}
$$

b. They are all in parallel :

$$
\begin{aligned}
& t_{\mathrm{F}}: \mathcal{M}(\mathrm{F})=0 \wedge \forall_{x \leq Q} \mathcal{M}\left(p_{x \mathrm{ok}}\right)=1 \\
& t_{\mathrm{Cx}}: \mathcal{M}(\mathrm{F})=1 \wedge \forall_{x \leq Q} \mathcal{M}\left(p_{x \mathrm{ko}}\right)=r \\
& t_{\mathrm{F}}^{\prime}: \mathcal{M}(\overline{\mathrm{F}})=1 \wedge \forall_{x \leq Q} \mathcal{M}\left(p_{x \mathrm{ok}}\right)=1 \\
& t_{\mathrm{Cx}}^{\prime}: \mathcal{M}(\overline{\mathrm{F}})=0 \wedge \forall_{x \leq Q} \mathcal{M}\left(p_{x \mathrm{ko}}\right)=r
\end{aligned}
$$

c. There are $\mathrm{Q}$ in parallel and $\mathrm{R}$ in series: In this case, we have to seperate them, obtaining the case presented in i.c. 


\section{Refinement of the Structural Model}

We assume that the structural model is organized in a modular manner, i.e., it is composed of sub-models representing the behavior of the system's components and their interactions. For several reasons, the first model that is built, starting from the functional-level model, may be not very detailed. One of these reasons could be the lack of information in the early system's selection and development phases. Another reason could be the complexity of the system to be modeled. To master this complexity a high-level model is built and then refined progressively.

As soon as more detailed information is available concerning the system's composition and events governing component evolution, the structural model can be refined.

Another refinement may be done regarding event distributions. Indeed, an assumption is made that all events governing the system's behavior are exponentially distributed, which, in some cases, is not a good assumption. In particular, failure rates of some components may increase over time.

Model refinement allows detailed behavior to be taken into account and leads to more detailed results compared to those obtained from a high-level model. In turn, these detailed results may help in selecting alternative solutions for a given structure. For our purpose, we consider three types of refinement: Component, state/event and distribution. Given the fact that the system's model is modular, refinement of a component's behavior is undertaken within the component's sub-model and special attention should be paid to its interactions with the other sub-models. However, we will mainly address the new dependencies created by the refinement, without discussing those already existing. The latter are either unchanged or should be refined according to the type of refinement achieved.

Component refinement consists in replacing a component by two or more components. From a modeling point of view, such a refinement leads to the transformation of the component's sub-model into another sub-model. Our approach is to use the same transformation rules as those used for the interface model presented in Section 3.

State/event fine-tuning consists in replacing, by a subnet, the place/transition corresponding to this state/event. We define basic refinement cases, whose combination covers most usual possibilities of state/event refinement.

For distribution adjustment, we use the method of stages. Considering an event whose distribution is to be transformed into a non-exponential one, this method consists in replacing the transition associated with this event, by a subnet simulating an increasing event rate. We have adapted already published work 
to take into account dependencies between the component under consideration and components with which it interacts. This is done without changing the sub-models of the latters.

A section is devoted to each refinement type.

\subsection{Component Decomposition}

Consider a single function achieved by a single software component on a single hardware component. Suppose that the software is itself composed of $\mathrm{N}$ components. Three basic possibilities are taken into account (combinations of these three cases allow modeling of any kind of system):

- The $\mathrm{N}$ components are in series;

- The $\mathrm{N}$ components are redundant, which means that they are structurally in parallel;

- There are $\mathrm{Q}$ components in parallel and $\mathrm{R}+1$ components in series (with $\mathrm{Q}+\mathrm{R}=\mathrm{N}$ ).

These decompositions are respectively called parallel, series and mixed. Our goal is to use refinement rules identical, as far as possible, to the ones used in Section 3.

In the following, we explain how a single component is replaced by its $\mathrm{N}$ components.

Series Decomposition Consider the decomposition of software S into two series components $\mathrm{S} 1$ and S2. This case is presented in Fig. 7.

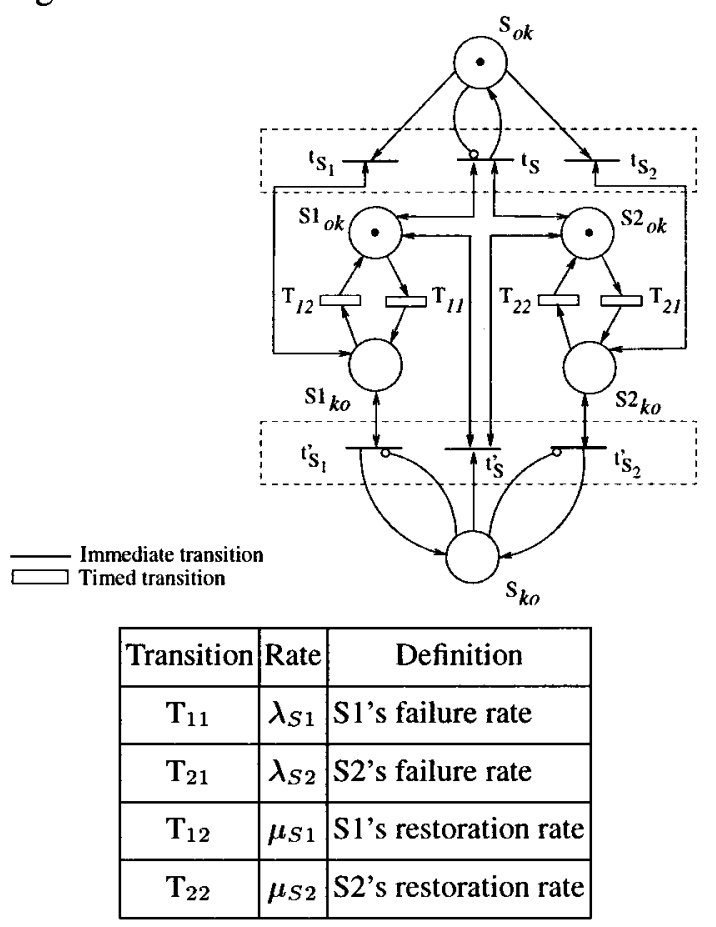

Fig. 7. Series decomposition 
Parallel Decomposition Consider software S's decomposition into two redundant components S1 and S2. Thus, S's up state is the result of S1 or S2's up states, and S's failure state is the combined result of S1 and S2's failure states.

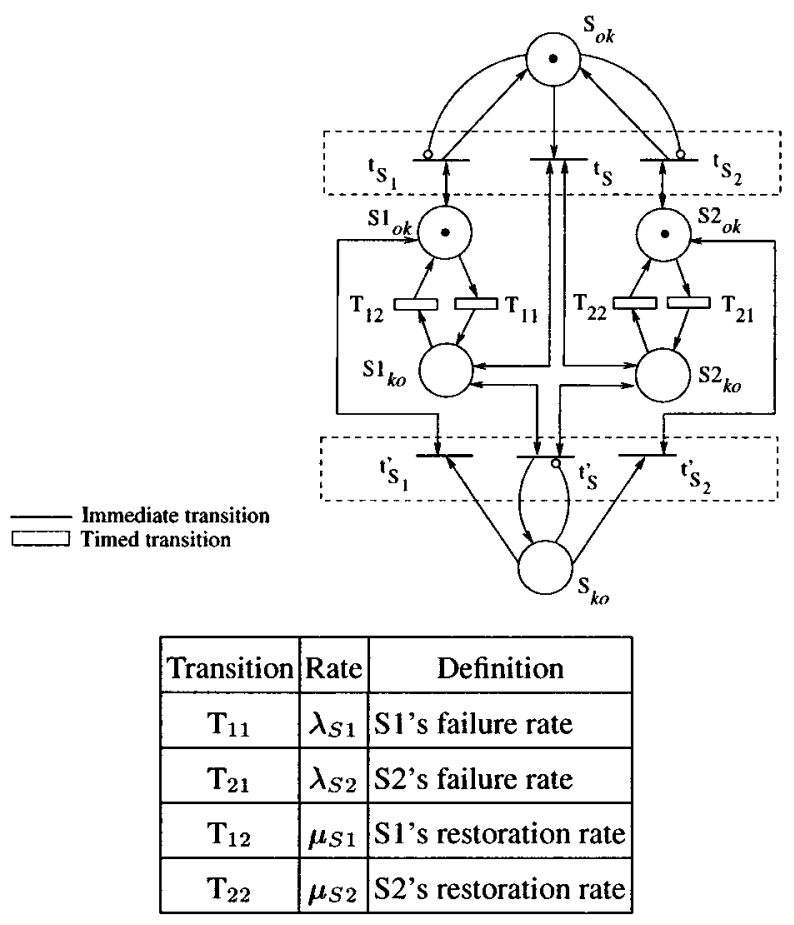

Fig. 8. Parallel decomposition

Fig. 8 gives a GSPN model of this case. The generalization to $\mathrm{N}$ components is straightforward.

Mixed Decomposition Suppose S composed of three components: S1, S2 and S3, where S3 is in series with S1 and S2, that are redundant. This case is identical to the example presented in Fig. 5 when replacing $\mathrm{F}$ by $\mathrm{S}$ and $\mathrm{H}$ by $\mathrm{S} 3$.

Conclusion It is worth mentioning that the interface model between the system and its components is built exactly in the same manner as the interface model between a function and its associated components. In all the cases illustrated above, we have considered only one token in each initial place. Indeed, $\mathrm{K}$ identical components can be modeled by a simple model with $\mathrm{K}$ tokens in each initial place. When refining the behavior of such components, a dissymmetry in their behavior may appear. This is due to the fact that some components that have the same behavior at a given abstraction level, may exhibit a slightly different behavior when more details are taken into account. If this is the case, one has to modify the model of the current abstraction level before refinement. This may lead to changing the interface 
model either between the functional-level and the structural model, or between two successive structural models. This is the only case where refinement leads to changing the model at the higher level.

\subsection{State/Event Fine-tuning}

In GSPNs, places correspond to system's states and timed transitions to events that guide state changes. The fine-tuning of places/transitions allows more detailed behavior to be modeled. In fact, state/event fine-tuning may result from assumptions' refinement or lead to a refinement of the assumptions. Refinement has been studied in Petri nets $([16,15])$ and more recently in Time Petri nets [11]. One of the objectifs of these refinement theories was to preserve the results of model processing.

Our goal is to detail the system's behavior by refining the underlying GSPN. Our sole constraint is to ensure that the net's dynamic properties (liveness, boundness and safeness), at each refinement step, are preserved. Our main motivation for model refinement is to have more detailed results about the system's behavior, that better reflect reality.

We define three basic refinement cases. Combinations of these three cases cover most usual situations for dependability models' refinement. They are given in Table 1.

Table 1. State/Event refinement

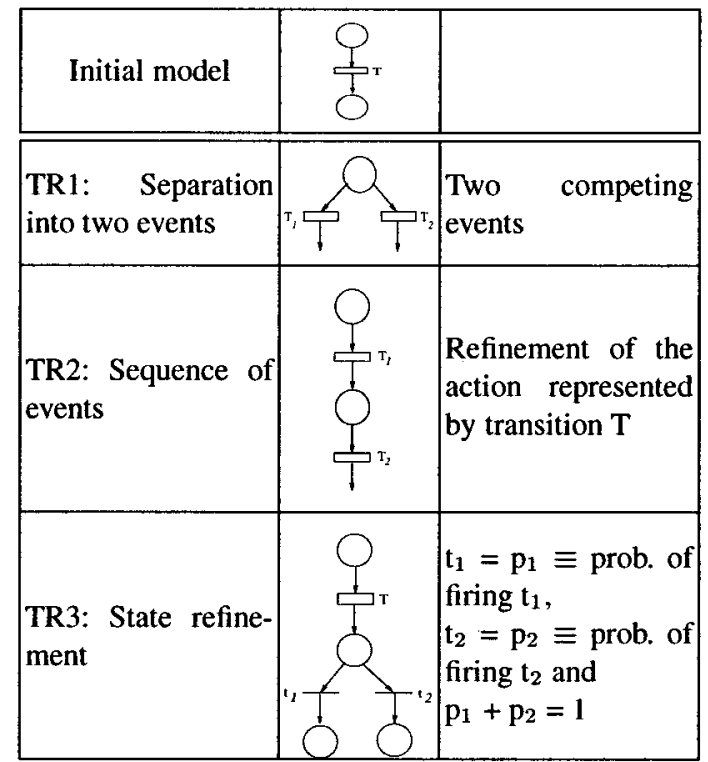

TR1 allows the replacement of one event by two competing events. It allows the event's separation into two other events with different rates. TR2 allows a sequential refinement of events, while TR3 allows the refinement of a state into two or more states. These transformations are illustrated on the following simple example. 
Consider the hardware model given in Fig. 9(a). Several successive refinement steps are depicted in Fig. 9(b), (c) and (d).

(a) Compact model

$\mathrm{T}_{1}=\lambda \equiv$ failure rate

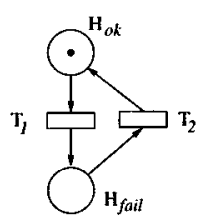

$\stackrel{\text { TR3 }}{\longrightarrow}$
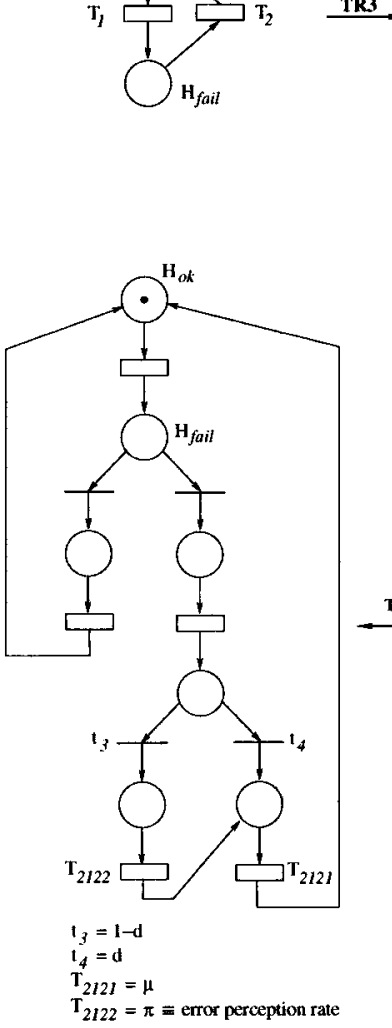

(d) Error detection efficiency (b) 2 types of faults

$\mathrm{t}_{1}=1-\mathrm{a}$

$\mathrm{t}_{2}=\mathrm{a}$
$\mathrm{T}_{21}=\mu \equiv$ repair rate

$T_{22}=\varepsilon \equiv$ disappearance rate

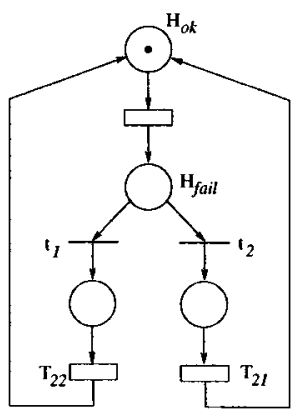

TR3

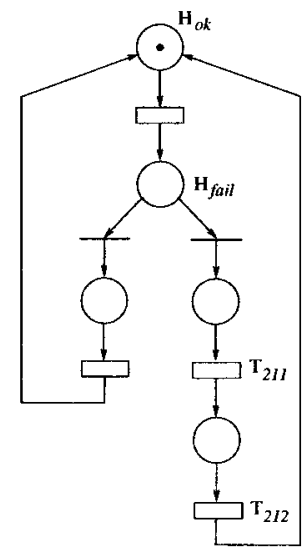

$\mathrm{T}_{211}=\delta \equiv$ error detection rate

$\mathrm{T}_{212}=\mu$

(c) Error detection latency

Fig. 9. State/Event refinement

After a fault activation $\left(T_{1}\right)$ two types of faults are distinguished: Temporary and permanent, with probability $a$ and $1-a$ respectively. Using TR3, we obtain the model depicted in Fig. 9(b). This corresponds to an assumption refinement.

To take into account error detection latency $(1 / \delta)$ for hardware components, we apply TR2 to transition $T_{21}$ of Fig. 9(b). The resulting model is presented in Fig. 9(c), wich is a state refinement.

Finally, we model the error detection efficiency by applying TR3. Detected errors allow immediate system's repair. We then add a perception latency (transition $T_{2122}$ ), Fig. $9(\mathrm{~d})$. This latency is important 
to be modeled because, as long as the effects of the non-detected error are not perceived, the system is in a non-safe state. Repair can be performed only after perception of the effects of such errors.

This is a small example of a state/event refinement application. Other details can be added to the model using the three rules presented in this section.

\subsection{Distribution Adjustment}

It is well known that the exponential distribution assumption is not appropriate for all event rates. For example, due to error conditions accumulating with time and use, the failure rate of a software component might increase.

The possibility of including timed transitions with non-exponential firing time is provided by the method of stages [10]. This method transforms a non Markovian process into a Markovian one, by decomposing a state (with a non-exponential firing time distribution) into a series of $k$ successive states. Each of these $k$ states will then have an exponential firing time distribution, to simulate an increasing rate. In GSPNs, a transition, referred to as extended transition, is replaced by a subnet to model the $k$ stages.

The transformation of an exponential distribution into a non-exponential one might create new timing dependencies. Indeed, the occurrence of some events in other components might affect the extended transition. For example, the restart of another software component might lead to the restart of the component under consideration (that has an increasing failure rate) and thus stop the accumulation of error conditions, bringing back the software under consideration to its initial state.

In previously published work $[1,2]$, the dependency between events is modeled only by concurrent transitions enabled by the same place. This is not very convenient when several components interact with the component under consideration, as it could lead to changing their models. We have adapted this extension method to allow more flexibility and to take into account this type of dependency.

The salient idea behind our approach is to refine the event's distribution without changing the submodels of the other components, whose behavior may affect the component under consideration (when assuming a non-exponential distribution).

In the rest of this section, we first present the extension method presented in [2] and then present our adapted extension method.

Previous Work Concerning the transitions' timers, three memory policies have been identified and studied in the literature, namely, resampling, age memory and enabling memory. The latter is well adapted to 
model the kind of dependency that is created when modeling system's dependability as mentioned above. It is defined as follows: At each transition firing, the timers of all the timed transitions that are disabled by this transition are restarted, whereas the timers of all the timed transitions that are not disabled hold their present values.

In [1] and [2] an application of the enabling memory policy in structural conflict situations has been given. It concerns the initial model of Fig. 10, in which transition $T_{1}$ to be extended is in structural conflict with transition $\mathrm{T}_{\text {res }}$.

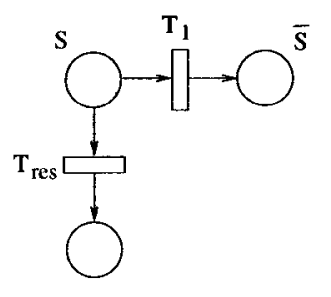

Fig. 10. Initial model

When applying the enabling memory policy as given in [2] to transition $T_{1}$ of Fig. 10, the resulting model is presented in Fig. 11. In this figure, the $k$ series stages are modeled by transitions $\mathrm{t}_{c 1}, \mathrm{t}_{c 2}, \mathrm{~T}_{1}^{1}$ and $T_{1}^{2}$ and places $P_{1}, P_{2}$ and $P_{3}$. Token moving in these places is controlled by the control places $P_{c 1}, P_{c 2}$ and $\mathrm{P}_{4} . \overline{\mathrm{S}}$ is marked once the $k$ stages have been crossed, through fire of $\mathrm{T}_{1}^{2}$.

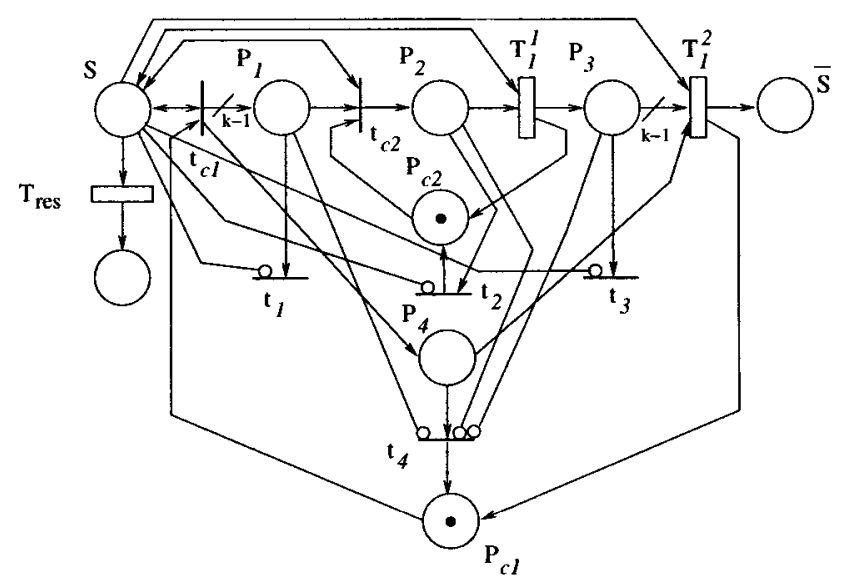

Fig. 11. Enabling memory with structural conflict

The enabling memory policy consists in stopping process $\mathrm{T}_{1}$ 's evolution after firing of $\mathrm{T}_{\text {res }}$ (i.e., clearing of places $\mathrm{P}_{1}, \mathrm{P}_{2}$ and $\mathrm{P}_{3}$ ). This is accomplished in two steps. As soon as $\mathrm{S}$ becomes empty, immediate transitions $\mathrm{t}_{1}, \mathrm{t}_{2}$ and $\mathrm{t}_{3}$ are fired as many times as needed to remove the $k$ tokens from places 
$P_{1}, P_{2}$ and $P_{3}$. At the end of this step, places $P_{c 2}$ and $P_{4}$ are marked with one token each. Once places $P_{1}$, $P_{2}$ and $P_{3}$ become empty, the return to the initial state is performed by immediate transition $t_{4}$ that puts one token in place $P_{c 1}$.

Enabling Memory with External Dependencies Our approach replaces the transition to be extended by two subnets: One internal to the component's model, to model its internal evolution, and a dependency subnet, that models its interaction with other components. The initial model is given in Fig. 12(a). In this model we assume that $T_{1}, T_{d i s 1}$ and $T_{d i s 2}$ are exponentially distributed. Suppose that in refining $\mathrm{T}_{1}$ 's distribution, its timer becomes dependent on $\mathrm{T}_{d i s 1}$ and $\mathrm{T}_{d i s 2}$. The transformed model is given in Fig. 12(b). A token is put in $\mathrm{P}_{d e p}$ each time the timer of transition $\mathrm{T}_{1}$ has to be restarted, due to the occurrence of an event that disables the event modeled by $\mathrm{T}_{1}$ (firing of $\mathrm{T}_{d i s 1}$ and $\mathrm{T}_{d i s 2}$ in other component models). Like in the previous case, this is done in two steps. As soon as place $\mathrm{P}_{d e p}$ is marked, $\mathbf{t}_{1}, \mathrm{t}_{2}{ }_{2}$ and $t^{\prime}{ }_{3}$ are fired as many times as needed to remove all tokens from places $P_{1}, P_{2}$ and $P_{3}$. Once places $P_{1}, P_{2}$ and $\mathrm{P}_{3}$ are empty, the return to the initial state is performed by transition $\mathbf{t}^{\prime}{ }_{4}$ that removes a token from place $P_{d e p}$ and puts one token in place $\mathbf{P}_{c 1}$.

Note that transitions $t^{\prime}{ }_{1}, t^{\prime}{ }_{2}, t^{\prime}{ }_{3}$ and $t^{\prime}{ }_{4}$ replace respectively $t_{1}, t_{2}, t_{3}$ and $t_{4}$. Also, we simplified Fig. 12(b), by replacing place $\mathrm{P}_{4}$ by an inhibitor arc between $\mathrm{t}_{4}$ and $\mathrm{P}_{c 1}$. Thus, the two major differences between Fig. 11 and 12(b) are: 1) Place $P_{1}$ of Fig. 12(b) is replaced by an inhibitor arc going from place

$\mathrm{P}_{c 1}$ to immediate transition $\left.t ; 2\right)$ Place $\mathrm{P}_{d e p}$, that manages dependencies between this net and the rest of the model, is added.

An example of application of this case is given in 6.

\section{Application to I\&C Systems}

In this section we illustrate the application of our modeling approach to I\&C systems. We first present the main functions together with the functional-level model for a general I\&C system. Then, we describe how the high-level dependability model is built for one of the I\&C systems. Finally, we show some results concerning a small part of its detailed dependability model.

\subsection{System Presentation}

An I\&C system performs five main functions: Human-machine interface (HMI), processing (PR), archiving (AR), management of configuration data (MD), and interface with other parts of the I\&C system (IP). 


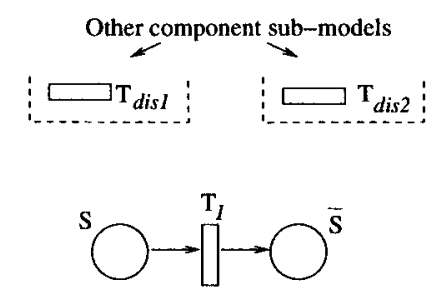

(a)

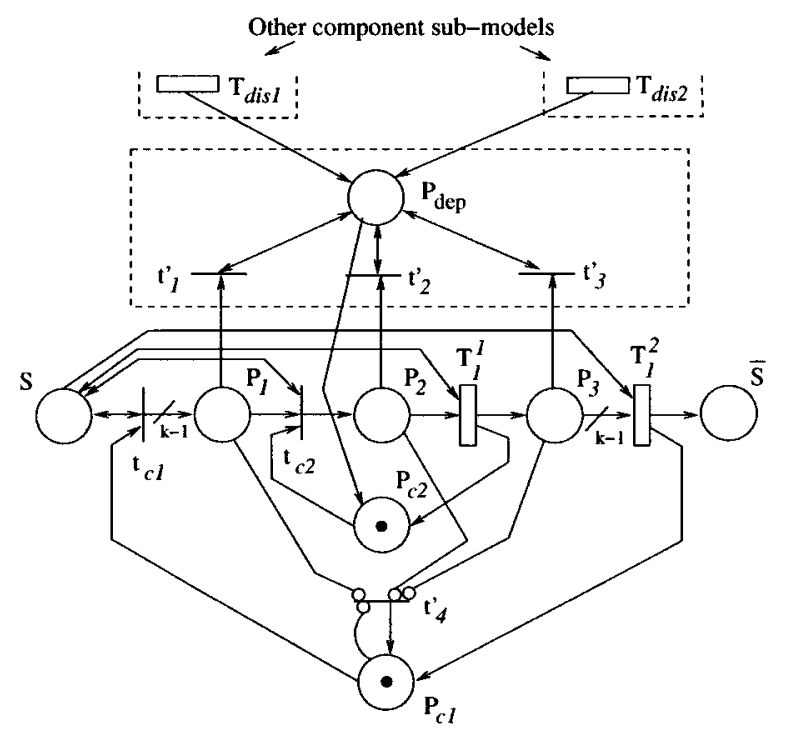

(b)

Fig. 12. Enabling memory with external dependencies

The functions are linked by the partial dependencies:

$$
\mathrm{HMI} \hookleftarrow\{\mathrm{AR}, \mathrm{MD}\}, \mathrm{PR} \hookleftarrow \mathrm{MD}, \mathrm{AR} \hookleftarrow \mathrm{MD} \text { and } \mathrm{IP} \hookleftarrow \mathrm{MD}
$$

These relations are modeled by the functional-level model depicted in Fig. 13.

To illustrate the second step of our modeling approach, we consider the example of an I\&C system composed of five nodes connected by a Local Area Network (LAN). The mapping between the various nodes and their functions is given in Fig. 14. Note that while HMI is executed on four nodes, Node 5 runs three functions. Nodes 1 to 4 are composed of one computer each. Node 5 is fault-tolerant: It is composed of two redundant computers. The initial structural model of this I\&C is built as follows:

- Node 1 to Node 3 - in each node, a single function is achieved by one software component on a hardware component. Its model is similar to the one presented in Fig. 4; 


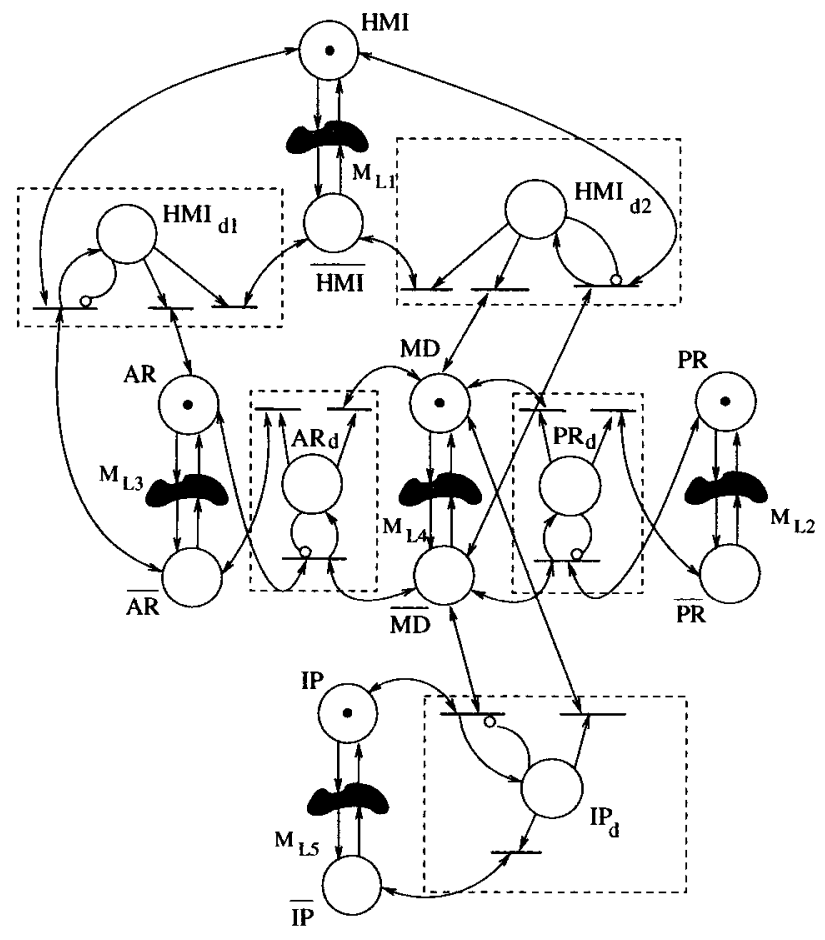

Fig. 13. Functional-level model for I\&C systems

- Node 4- has two functions that are partially dependent. Its functional-level model will be similar to $F_{1}$ and $F_{2}$ 's functional-level model given in Fig. 3. Its structural model will be similar to the one depicted in Fig. 6;

- Node 5 - is composed of two hardware components with three independent functions each. Its structural model is more complex. A part of it is given in Section 5.2;

- LAN - two different assumptions can be made: i) It is non fault tolerant, in which case its model is similar to the one presented in Fig. 4; ii) it is fault tolerant, its model will be a duplex one. Moreover, since the LAN is a single point of failure, it is in series with all of the other components. This means that the LAN's failure induces the loss of the system's functions. For this reason, its model is not presented in Fig. 13.

The complete high-level dependability model for this system is composed of 41 places and 19 tokens. After refinement, the model is much larger, as illustrated in Section 5.3.

In the rest of the section, we concentrate on a single function: Function AR of Node 5. We first present its high-level dependability model, and then we show how it can be refined progressively. We conclude the section with some dependability evaluation results. 


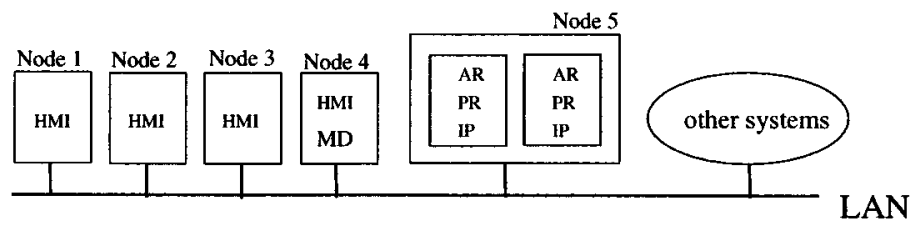

Fig. 14. I\&C structure

\subsection{AR High-level Dependability Model}

Let us consider the case of Node 5 of Fig. 14. Function AR is performed by two units ${ }^{1}$ : A primary unit and a secondary one. When the primary fails, a switch between the two units is attemped. The global view for the high-level dependability model of function AR is presented in Fig. 15. In this figure, SH, PH, SS and PS correspond respectively, to the models of the secondary hardware component, primary hardware component, secondary software component and primary software component. Arrows from SH to SS, and from PH to PS represent in each case, the software component's stop when the respective hardware component fails. This will be detailed later.

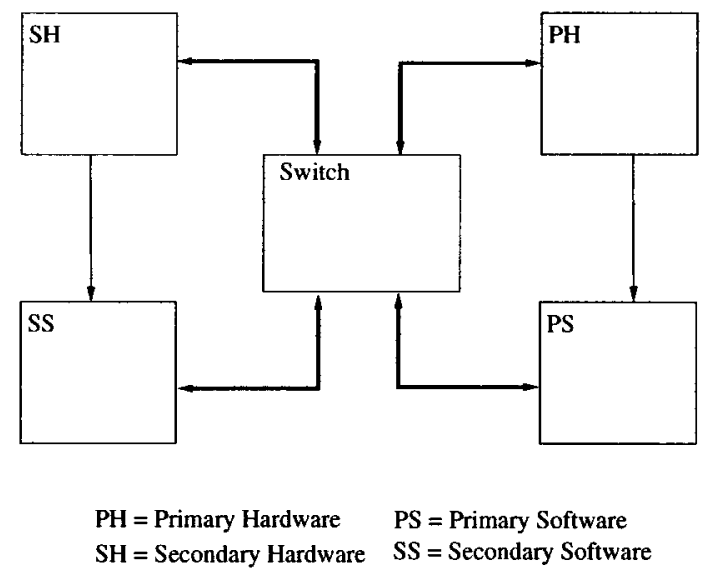

Fig. 15. High-level dependability modules for function AR

The high-level GSPN dependability model for the AR function is given in Fig. 16. In this figure, each set $\{\mathrm{SH}, \mathrm{SS}\}$ and $\{\mathrm{PH}, \mathrm{PS}\}$, corresponds to the complete high-level model of Fig. 4. It is worth noting that we consider two software unavailability states: $S_{1 s t}$ and $S_{1 \text { fail }}$. The first one corresponds to the software's stop after its correspondant hardware failure. $S_{1 \text { fail }}$ corresponds to the software failure state. To the complete model of Fig. 4, we added the switch module which is composed of place $\mathbf{P}_{s w}$

\footnotetext{
${ }^{1}$ A unit is composed of a hardware computer and its corresponding software component (at this level of detail, we suppose that each function is executed by a single software component).
} 
and timed transition $\mathrm{T}_{s w}$. If the switch succeeds (immediate transitions $\mathrm{t}_{s w s 1}$ and $\mathrm{t}_{s w s 2}$ ), the function continues to be executed. If it does not succeed (immediate transition $\mathrm{t}_{s w f}$ ), the function fails.

It is worth noting that the switch may be activated following a software or a hardware failure. In either case, it switches simultaneously hardware and software components.

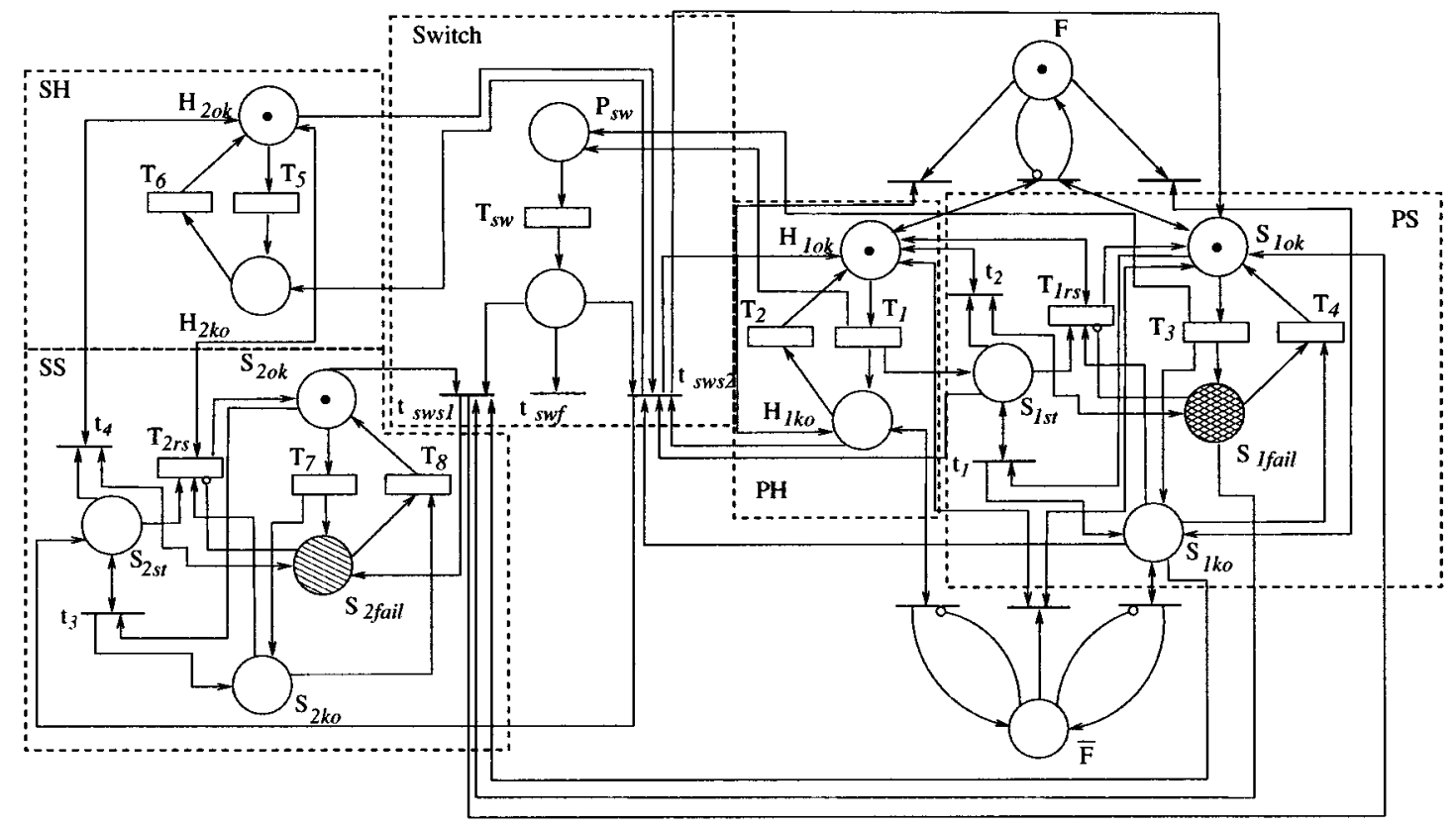

Fig. 16. High-level dependability model for function AR

\subsection{AR Model Refinement}

Once the model is refined, the modules obtained are given in Fig. 17. In this figure, $\mathrm{SH}_{r f}, \mathrm{PH}_{r f}, \mathrm{SS}_{r f}$ and $\mathrm{PS}_{r f}$ correspond respectively, to the refined models of the secondary hardware component, primary hardware component, secondary software component and primary software component. It is worth noting that refinement introduces new dependencies between components: In this case, modules $\mathrm{EP}_{s h s}, \mathrm{EP}_{p h s}$ and $\mathrm{EP}_{s s}$, corresponding to the error propagation between components. This is due to the refinement of the modeling assumptions. Indeed, Fig. 17 distinguishes permanent and temporary errors (wich is not the case for Fig. 15). As a consequence, Fig. 17 assumes that temporary hardware errors might propagate to the software $\left(\mathrm{EP}_{p h s}\right.$ and $\left.\mathrm{EP}_{s h s}\right)$ and temporary software errors of the primary might propagate to the secondary software $\left(\mathrm{EP}_{s s}\right)$

In Fig. 17, the switch model is the same as in Fig. 15. This is true when assuming the same switch rate for hardware and software components, that is, $1 / \beta_{h}=1 / \beta_{s}$. However, if we consider that these values 


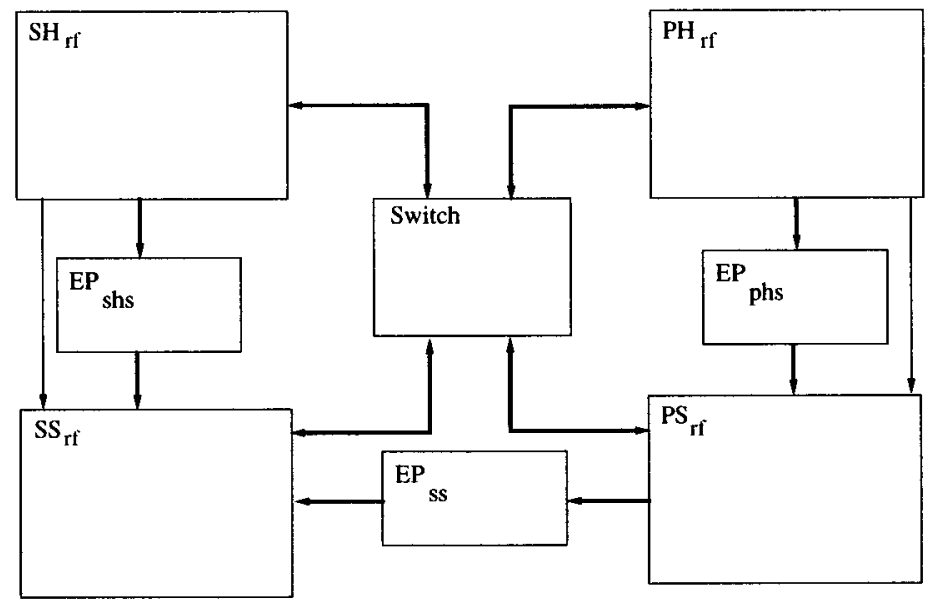

$\mathrm{EP}_{\text {shs }}=$ Secondary hardware-software error propagation
$\mathrm{EP}_{\text {phs }}=$ Primary hardware-software error propagation
$\mathrm{EP}_{\mathrm{ss}}=$ Software-software error propagation

Fig. 17. Detailed dependability modules for function AR

are different, when refining the models, we obtain two switch models: One corresponding to the switch between hardware components and the other to the switch between software components.

A part of the associated detailed structural model is given in Fig. 18 in which the $S_{1 \text { fail }}$ place of Fig. 16, corresponds to either place $S_{r s p}$ or $S_{r i p}$, and place $S_{2 f a i l}$ to either place $S_{r s s}$ or $S_{r i s}$.

Note that a software fault may propagate from the primary to the secondary with probability $p_{s s}$.

The detailed GSPNs presented are obtained using the rules described in Section 4.2. The following assumptions and notations are used (Fig. 18):

- The activation rate of a software fault on the primary is $\lambda_{s p}\left(\mathrm{~T}_{a f s p}\right)$ and of $\lambda_{s s}\left(\mathrm{~T}_{a f s s}\right)$ on the secondary;

- A software fault is detected by the fault tolerant mechanisms with probability $d_{s}$. The detection rate is $\delta_{s}\left(\mathrm{~T}_{\text {desp }} / \mathrm{T}_{\text {dess }}\right)$;

- The effects of a non detected error are perceived with rate $\pi_{s}\left(\mathrm{~T}_{e p s p}\right)$;

- Errors in the software may necessitate only a reset. The reset rate is $\rho\left(\mathrm{T}_{r s p} / \mathrm{T}_{r s s}\right)$ and the probability that an error induced by the activation of a software fault disappears with a reset is $r\left(t_{r s f p} / t_{r s r s}\right)$;

- If the error does not disappear with the software reset, the software is re-installed. The software's re-installation rate is $\sigma\left(\mathrm{T}_{\text {rip }} / \mathrm{T}_{\text {ris }}\right)$.

The following assumptions and notations are used (Fig. 19):

- The activation rate of a hardware fault is $\lambda_{h}\left(\mathrm{~T}_{a f h p}\right)$; 


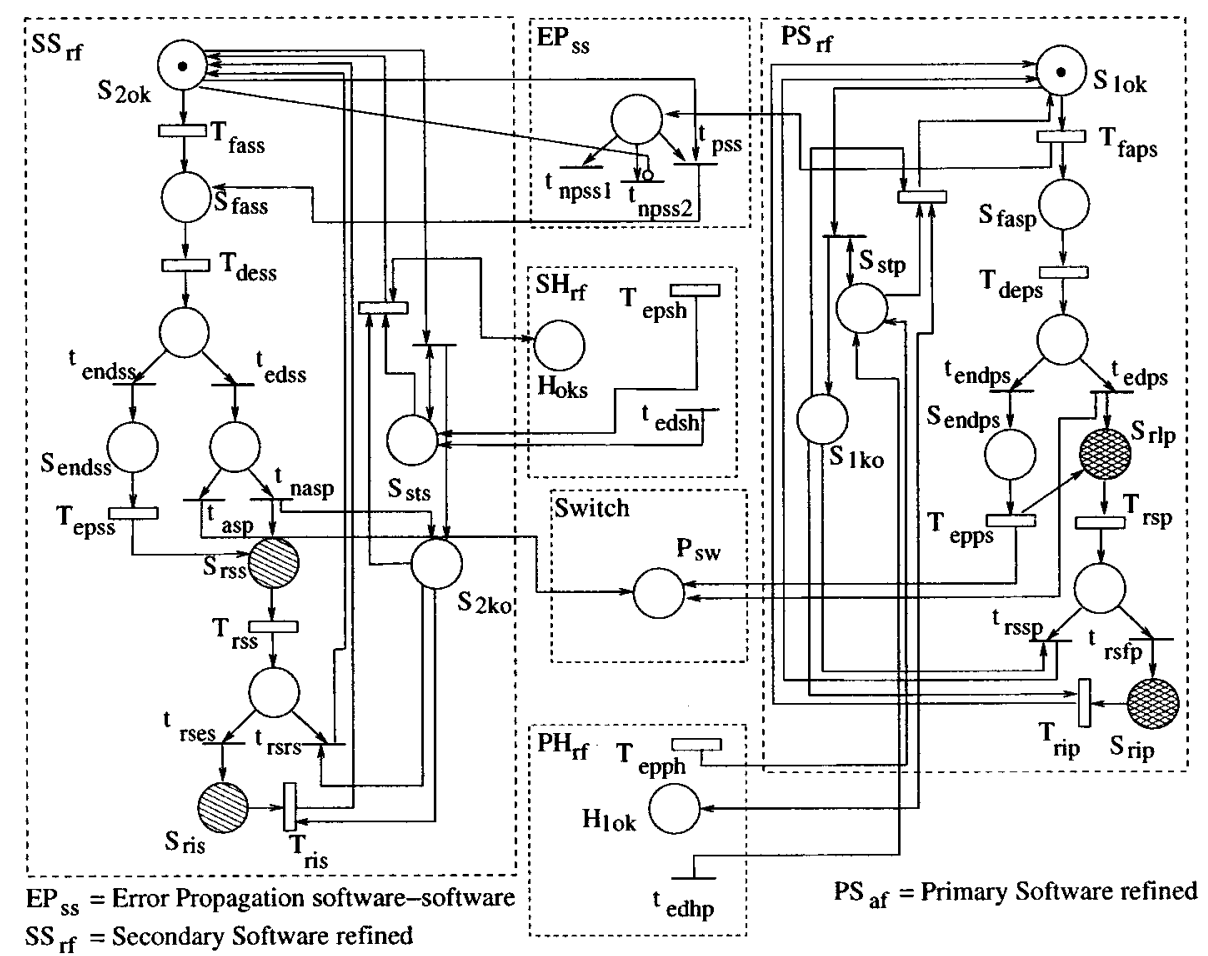

\begin{tabular}{|c|l|}
\hline Place & \multicolumn{1}{|c|}{ Definition } \\
\hline $\mathrm{S}_{1 o k} / \mathrm{S}_{2 o k}$ & primary/secondary software's up state \\
\hline $\mathrm{S}_{f a s p} / \mathrm{S}_{f a s s}$ & activate fault on the primary/secondary software \\
\hline $\mathrm{S}_{e n d s p} / \mathrm{S}_{e n d s s}$ & primary/secondary non-detected error \\
\hline $\mathrm{S}_{r s p} / \mathrm{S}_{r s s}$ & restart of the primary/secondary software \\
\hline $\mathrm{S}_{r i p} / \mathrm{S}_{r i s}$ & re-installation of the primary/secondary software \\
\hline $\mathrm{S}_{1 k o} / \mathrm{S}_{2 k o}$ & primary/secondary software's down state \\
\hline $\mathrm{P}_{s w}$ & switch between units \\
\hline
\end{tabular}

\begin{tabular}{|c|c|l|}
\hline Transition & Rate & \multicolumn{1}{|c|}{ Definition } \\
\hline $\mathrm{T}_{a f s p}$ & $\lambda_{p s}$ & primary's fault activated \\
\hline $\mathrm{T}_{a f s s}$ & $\lambda_{s s}$ & secondary's fault activated \\
\hline $\mathrm{T}_{\text {desp }} / \mathrm{T}_{\text {dess }}$ & $\delta_{s}$ & software error detection on the primary/secondary \\
\hline $\mathrm{T}_{\text {epsp }} / \mathrm{T}_{\text {epss }}$ & $\pi_{s}$ & primary/sedondary software error perception \\
\hline $\mathrm{T}_{r s p} / \mathrm{T}_{r s s}$ & $\rho$ & primary/secondary software restart \\
\hline $\mathrm{T}_{r i p} / \mathrm{T}_{r i s}$ & $\sigma$ & primary/secondary software re-installation \\
\hline
\end{tabular}

Fig. 18. Software redundant module with error propagation 


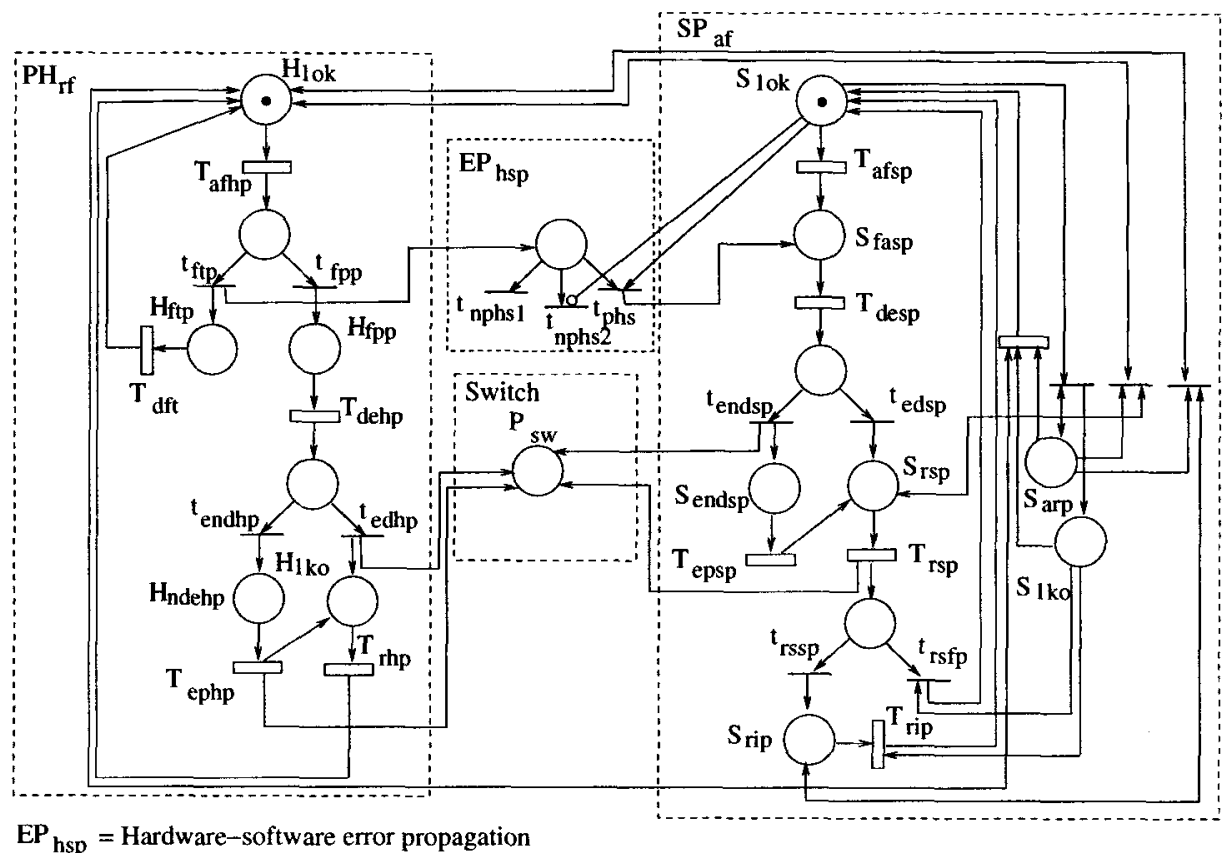

$\mathrm{EP}_{\mathrm{hsp}}=$ Hardware-software error propagation

\begin{tabular}{|l|l|}
\hline Place & \multicolumn{1}{|c|}{ Definition } \\
\hline $\mathrm{H}_{1 o k}$ & hardware's up state \\
\hline $\mathrm{H}_{f t p}$ & hardware' temporary fault \\
\hline $\mathrm{H}_{f p p}$ & hardware' permanent fault \\
\hline $\mathrm{H}_{n d e h p}$ & non-detected error \\
\hline $\mathrm{H}_{1 k o}$ & hardware's down state \\
\hline $\mathrm{P}_{s w}$ & switch between units \\
\hline
\end{tabular}

\begin{tabular}{|c|c|l|}
\hline Transition & Rate & \multicolumn{1}{|c|}{ Definition } \\
\hline $\mathrm{T}_{a f s p}$ & $\lambda_{p s}$ & primary's fault activated \\
\hline $\mathrm{T}_{a f s s}$ & $\lambda_{s s}$ & secondary's fault activated \\
\hline $\mathrm{T}_{\text {desp }} / \mathrm{T}_{\text {dess }}$ & $\delta_{s}$ & software error detection on the primary/secondary \\
\hline $\mathrm{T}_{e p s p} / \mathrm{T}_{e p s s}$ & $\pi_{s}$ & primary/sedondary software error perception \\
\hline $\mathrm{T}_{r s p} / \mathrm{T}_{r s s}$ & $\rho$ & primary/secondary software restart \\
\hline $\mathrm{T}_{r i p} / \mathrm{T}_{r i s}$ & $\sigma$ & primary/secondary software re-installation \\
\hline
\end{tabular}

Fig. 19. Hardware and software module with error propagation 
- The probability that a hardware fault is temporary is $t\left(\mathrm{t}_{f t p}\right)$. Such faults will disappear with rate $\varepsilon$ $\left(\mathrm{T}_{d f t}\right)$;

- A permanent hardware fault is detected by the fault-tolerance mechanisms with probability $d_{h}$. The detection rate is $\delta_{h}\left(\mathrm{~T}_{\text {dehp }}\right)$ for the hardware;

- The effects of a non detected error are perceived with rate $\pi_{h}\left(\mathrm{~T}_{e p h p}\right)$;

- Errors detected in the hardware component require its repair: Repair rate is $\mu\left(\mathrm{T}_{r h p}\right)$.

Note that a temporary fault in the hardware may propagate to the software $\left(\mathrm{t}_{p s s}\right)$ with probability $p_{h s}$.

Also, when the hardware is in the repair state, the software is on hold. The software will be reset or re-installed as soon as the hardware repair is finished.

\subsection{AR Availability Evaluation}

The model has been processed using the SURF-2 tool [7]. The unavailability, evaluated in hours per year according to the switch parameters (switching time $1 / \beta$ and coverage factor ${ }^{2} c$ ) is given in table 2 .

It can be seen that both parameters have significant impact. It is obvious that the smallest unavailability is obtained for the shortest switch time and the best coverage factor. This table shows that an annual unavailability of approximately $2 \mathrm{~h}$ may be obtained for $(0.99 ; 5 \mathrm{~min})$ and $(0.98 ; 1 \mathrm{~min}$ or $30 \mathrm{~s})$.

Table 2. AR annual unavailability: $1 / \lambda_{h}=1000 \mathrm{~h} 1 / \mu_{h}=10 \mathrm{~h}$

\begin{tabular}{ccccc}
\hline$c \backslash 1 / \beta$ & $30 \mathrm{~s}$ & $1 \mathrm{~min}$ & $5 \mathrm{~min}$ & $10 \mathrm{~min}$ \\
\hline 0.99 & $\mathbf{1 h 3 4 m i n}$ & $\mathbf{1 h 3 8 m i n}$ & $\mathbf{2 h 0 6 m i n}$ & $2 \mathrm{~h} 45 \mathrm{~min}$ \\
0.98 & $\mathbf{2 h 0 0 m i n}$ & $\mathbf{2 h 0 4 m i n}$ & $2 \mathrm{~h} 33 \mathrm{~min}$ & 3h11 min \\
0.95 & 3h20min & 3h23min & 3h52min & 4h31min \\
\hline
\end{tabular}

A second example of results (table 3 ) shows how the annual unavailability is affected by the variation of the hardware failure rate value.

The only values of $1 / \beta$ and $c$ leading to an annual unavailability of 2 hours are $1 / \beta \leq 1 \mathrm{~min}$ and $c=0.95$. Also, there is a difference of about 22 minutes, and even more than an hour in case of $(0.95$; $10 \mathrm{~min})$.

The last result example presented here concerns a change in the repair time. Table 4 shows that reducing the hardware repair duration from $10 \mathrm{~h}$ to $2 \mathrm{~h}$, does not improve significantly the annual system's availability (compared to table 2 .

${ }^{2}$ Probability that the switch succeeds. 
Table 3. Node 5 annual unavailability: $1 / \lambda_{h}=100 \mathrm{~h} 1 / \mu_{h}=10 \mathrm{~h}$

\begin{tabular}{ccccc}
\hline$c \backslash 1 / \beta$ & $30 \mathrm{~s}$ & $1 \mathrm{~min}$ & $5 \mathrm{~min}$ & $10 \mathrm{~min}$ \\
\hline 0.99 & $\mathbf{1 h} 56 \mathrm{~min}$ & $\mathbf{2 h} 00 \mathrm{~min}$ & $2 \mathrm{~h} 36 \mathrm{~min}$ & $3 \mathrm{~h} 26 \mathrm{~min}$ \\
0.98 & 2h29min & 2h33min & $3 \mathrm{~h} 10 \mathrm{~min}$ & $3 \mathrm{~h} 59 \mathrm{~min}$ \\
0.95 & 4h09min & 4h13min & 4h50min & $5 \mathrm{~h} 39 \mathrm{~min}$ \\
\hline
\end{tabular}

Table 4. AR annual unavailability $1 / \lambda_{h}=1000 \mathrm{~h} 1 / \mu_{h}=2 \mathrm{~h}$

\begin{tabular}{ccccc}
\hline$c \backslash 1 / \beta$ & $30 \mathrm{~s}$ & $1 \mathrm{~min}$ & $5 \mathrm{~min}$ & $10 \mathrm{~min}$ \\
\hline 0.99 & $\mathbf{1 h 3 3 m i n}$ & $\mathbf{1 h 3 7 m i n}$ & $\mathbf{2 h 0 5 m i n}$ & 2h44min \\
0.98 & $\mathbf{2 h 0 0 m i n}$ & $\mathbf{2 h 0 3 m i n}$ & 2h32min & 3h10min \\
0.95 & 3h19min & 3h23min & 3h51min & 4h30min \\
\hline
\end{tabular}

\section{Conclusions}

Our modeling approach follows in the footsteps of most of the existing work on dependability modeling. Where this approach is unique is in the inclusion of the system's functional specifications into the dependability model, by means of a functional-level model. Also, it allows modeling of one system from its functional specification up to its implementation. The existing refinement techniques are conceived in order to preserve the result values. On the contrary, ours provides more accurate models and associated results.

Thus, the modeling approach presented in this papers gives a generally-applicable process for system's analysis, based on generalized stochastic Petri nets. This process involves a stepwise refinement in which dependencies are introduced at the appropriate level of refinement. A careful and precise definition of the constructs and of the refinement process is given. Indeed, we have shown how starting from functional specifications, a functional-level model can be transformed progressively into a dependability model taking into account the system's structure. We have also shown how the structural model can be refined to incorporate more detailed information of the system's behavior. Refinement is a very powerful tool for mastering progressively model construction. It will allow experimented, but not necessarily specially-trained, modelers to analyze the dependability of one or several systems and compare their dependability at the same level of modeling abstraction, if required. 
The approach was illustrated here on simple examples related to a specific structure of an instrumentation and control system in power plants. However, we have applied this approach to three different I\&C systems to identify their strong and weak points, in order to select the most appropriate one [6].

\section{References}

1. M.A. Ajmone Marsan and G. Chiola. On Petri Nets with Deterministic and Exponentially Distributed Firing Time. LNCS 266:132-145, 1987.

2. M.A. Ajmone Marsan, G. Balbo, G. Conte, S. Donatelli and G. Franchescinis. Modelling with Generalized Stochastic Petri Nets. Series in Parallel Computing, Wiley, 1995.

3. C. Betous-Almeida and K. Kanoun. Dependability Evaluation: From Functional to Structural Modelling. Proc. 20th Int. Conf. on Computer Safety, Reliability and Security (SAFECOMP 2001), LNCS 2187:227-237, 2001.

4. C. Betous-Almeida. Construction and Refinement of Dependability Models - Application to Instrumentation and Control Systems. PhD Dissertation (in French). LAAS-CNRS Repport 02275, 2002.

5. C. Betous-Almeida and K. Kanoun. Stepwise Construction and Refinement of Dependability Models. Proc. of the International Conference on Dependable Systems and Networks (IPDS), 515-524, 2002.

6. C. Betous-Almeida and K. Kanoun. Dependability Modelling of Instrumentation and Control Systems: A Comparison of Competing Architectures. LAAS-CNRS Repport 02204, 2002.

7. C. Béounes and al. SURF-2: A Program for Dependability Evaluation of Complex Hardware and Software Systems. Proc. 23rd. Int. Symp. on Fault-Tolerant Computing (FTCS-23), 668-673, 1993.

8. A. Bondavalli, I. Mura and K.S. Trivedi. Dependability Modelling and Sensitivity Analysis of Scheduled Maintenance Systems. Proc. 3rd European Dependable Computing Conf. (EDCC-3), LNCS 1667:7-23, 1999.

9. P. Chen, S.C. Bruell and G. Balbo. Alternative Methods for Incorporating Non-Exponential Distributions into Stochastic Timed Petri Net. Proc. of the 3rd Int. Workshop on Petri Nets and Performance Models (PNPM'89), IEEE Computer Society Press:187-197, Decembre 1989.

10. D.R. Cox and H.D. Miller. The Theory of Stochastic Processes. Chapman and Hall Ltd, 1965.

11. M. Felder, A. Gargantini and A. Morzenti. A Theory of Implementation and Refinement in Timed Petri Net. Theoretical Computer Science, 202(1-2):127-161, 1998.

12. N. Fota, M. Kaâniche and K. Kanoun. Incremental Approach for Building Stochastic Petri Nets for Dependability Modeling. Statistical and Probabilistic Models in Reliability (D. C. Ionescuand N. Limnios, Eds.):321-335, BirkhBduser, 1999.

13. K. Kanoun, M. Borrel, T. Morteveille and A. Peytavin. Availability of CAUTRA, a Subset of the French Air Traffic Control System. IEEE Trans. on Computers, 48(5):528-535, May 1999.

14. M. Rabah, and K. Kanoun. Dependability Evaluation of a Distributed Shared Memory Multiprocessor System. Proc. 3rd European Dependable Computing Conf. (EDCC-3), LNCS 1667:42-59, 1999.

15. I. Suzuki and T. Murata. A Method for Stepwise Refinement and Abstraction of Petri Net. Journal of Computer and System Sciences, 27:51-76, 1983.

16. R. Valette. Analysis of Petri Nets by Stepwise Refinement. Journal of Computer and System Sciences, 18(1):35-46, February 1979. 


\section{Annexe}

We present here an example of application of the enabling memory with external dependencies presented in Section 4.3.

Consider a fault tolerant system (two redundant computers: $\mathrm{C} 1$ and $\mathrm{C} 2$ and two redundant disks: D1 and D2) with two tasks of different priority (Fig. 20).

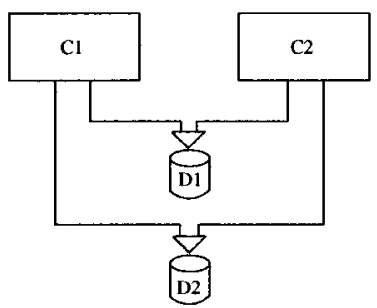

Fig. 20. Duplex system with two tasks of different priority

The system has the following characteristics: $\mathrm{C} 1$ executes high priority task HT, $\mathrm{C} 2$ executes low priority task LT, HT uses $\mathrm{C} 1$ and D1 or D2, and LT uses C2 and D1 or D2.

Under these conditions, if:

- C1 fails (and C2 is in its up state) - LT is stopped and HT is executed in C2;

- C2 fails (and C1 is in its up state) - LT is stopped and HT will continue to be executed in C1;

- D1 (or D2) fails - both tasks (HT and LT) will continue to be executed;

- D1 and D2 fail - both tasks are stopped.

Some precisions on the system's functionning in case of failure of one or more of its components:

- The transfert of HT to C2 is done by authomatic coverage by means of D1 or D2;

- The failure rate of each $\mathrm{C}_{i}$ is $\lambda$;

- There are two types of disk failure: Electronical (with rate $\lambda_{e}$ ) and electromecanical (with rate $\lambda_{m}$ );

- There is only one repairman;

- The repair rate of each $C_{i}$ is $\mu$;

- There are two types of disk repair: Electronical (with rate $\mu_{e}$ ) and electromecanical (with rate $\mu_{m}$ ). 


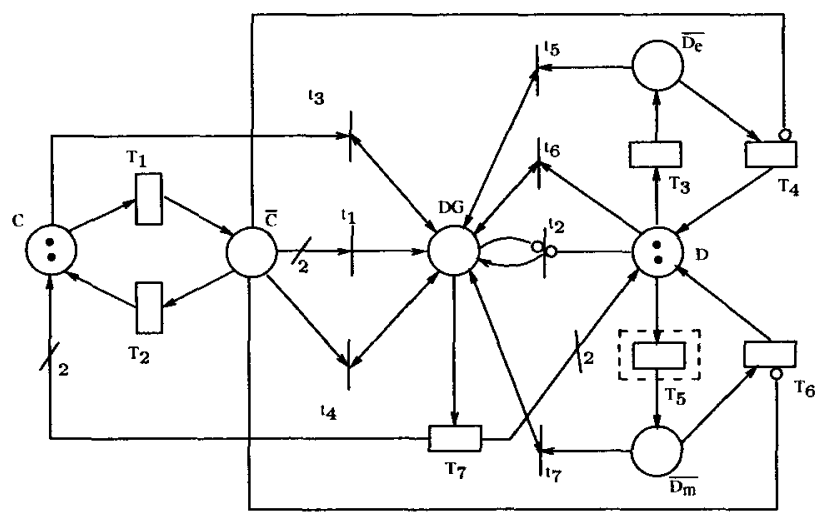

\begin{tabular}{|c|l|}
\hline Places & \multicolumn{1}{|c|}{ Definition } \\
\hline \hline C & C's nominal state \\
\hline$D$ & D's nominal state \\
\hline$\overline{\mathrm{C}}$ & C's fail state \\
\hline$\overline{\mathrm{D}} \overline{\mathrm{e}}$ & electronical D's fail state \\
\hline$\overline{\mathrm{D}_{\mathrm{m}}}$ & electromecanical D's fail state \\
\hline$\overline{D G}$ & global system's fail state \\
\hline
\end{tabular}

\begin{tabular}{|c|c|l|}
\hline Transitions & Rate & \multicolumn{1}{|c|}{ Definition } \\
\hline \hline$T_{1}$ & $\mathcal{M}(\mathrm{C}) \lambda$ & C failure activation \\
\hline $\mathrm{T}_{2}$ & $\mu$ & C's repair \\
\hline $\mathrm{T}_{3}$ & $\mathcal{M}(\mathrm{D}) \lambda_{e}$ & D's electronical failure \\
\hline $\mathrm{T}_{4}$ & $\mu_{e}$ & D's electronical repair \\
\hline $\mathrm{T}_{5}$ & $\mathcal{M}(\mathrm{D}) \lambda_{m}$ & D's electromecanical failure \\
\hline $\mathrm{T}_{6}$ & $\mu_{m}$ & D's electromecanical repair \\
\hline $\mathrm{T}_{7}$ & $\rho$ & global system's repair \\
\hline
\end{tabular}

$\mathcal{M}(\mathrm{D})$ corresponds to place D's marking

$\mathcal{M}(\mathrm{C})$ corresponds to place C's marking

Fig. 21. GSPN model of the duplex system

We assume that since there is only one repairman, both $\mathrm{C}_{i}$ have repair priority over each $\mathrm{D}_{i}$. Also, if both $C_{i}$ or both $D_{i}$ are in a failure state, there is a global system's stop and repair.

Fig. 21 gives the GSPN model of the system's behavior considering all the above given assumptions. The figure tables present the correspondance between the places/timed transitions with the system's states/transitions rates.

Immediate transitions $t_{3}, t_{4}, t_{5}, t_{6}$ and $t_{7}$ allow to empty places $C, \bar{C}, \overline{D_{e}}, D$ and $\overline{D_{m}}$ respectively, when a general system's failure arrises.

Assume that timed transition $\mathrm{T}_{5}$ 's law is an Erlang- $k$. In this case, when a global system's failure arrises, the system's developer must choose between the replacement of the fail component(s) or a global system's repair.

This last case corresponds to the enabling memory policy. Fig. 22 gives the correspondant GSPN model. It is worth noting that place DG, wich empties the subnet, is not the same as the one that ac- 
tivates/stops the timer (D place) of timed transition $\mathrm{T}_{5}$. Thus, DG place corresponds to place $\mathrm{P}_{d e p}$ and immediate transitions $t_{10}, t_{11}$ and $t_{12}$ correspond to immediate transitions $t^{\prime}{ }_{1}, t^{\prime}{ }_{2}$ et $t^{\prime}{ }_{3}$ of Fig. 12.

Thus, it is important when making a distribution modification, to take into account the existing structural conflicts and the possible time conflicts.

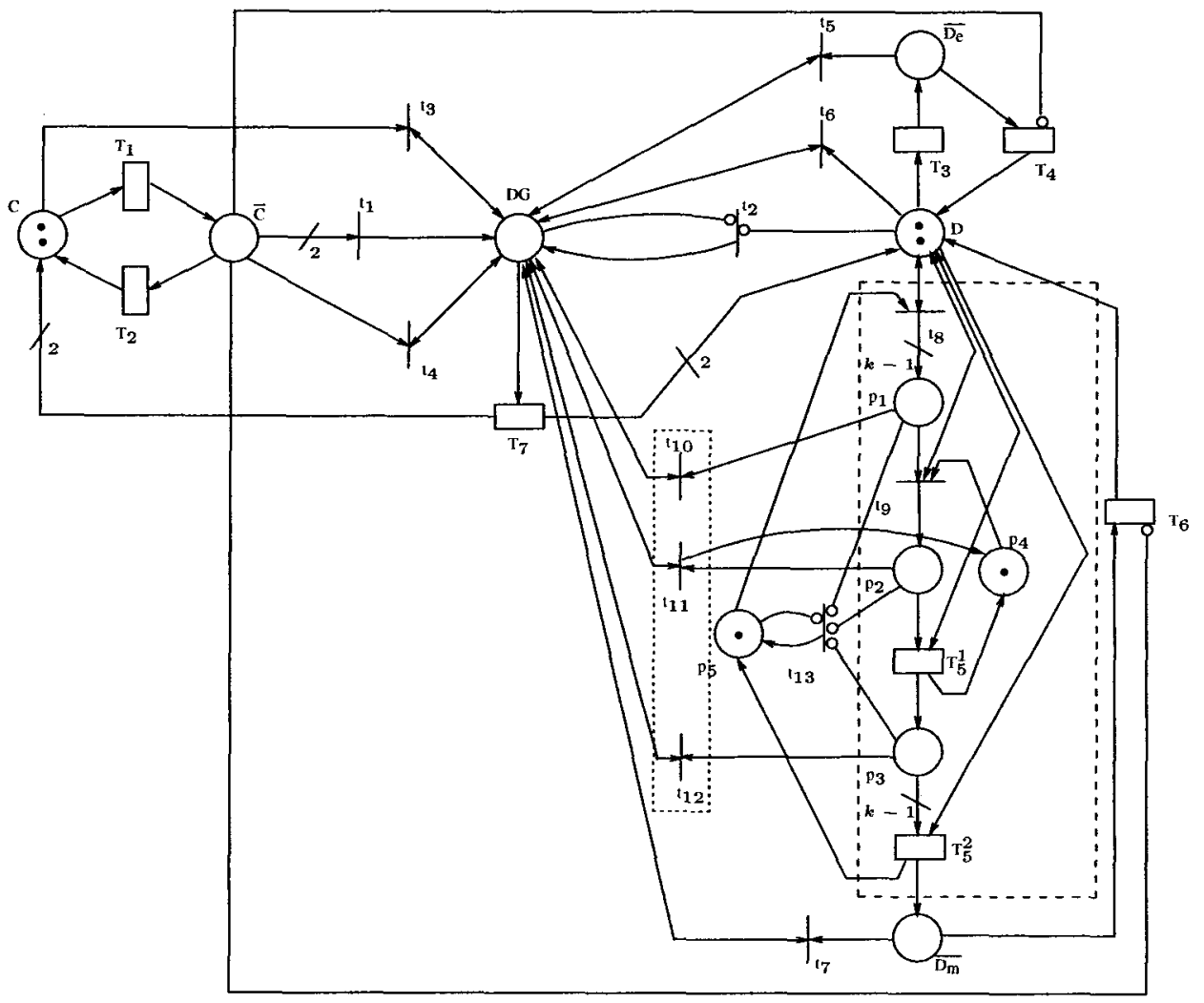

Fig. 22. GSPN model of the enabling memory case 